\title{
ANALYTICITY OF SOLUTIONS OF THE GENERALIZED KORTEWEG-DE VRIES EQUATION WITH RESPECT TO THEIR INITIAL VALUES
}

\author{
Bing-Yu Zhang *广 \\ Institute of Mathematics and Its Applications \\ University of Minnesota \\ Minneapolis, Minnesota 55455
}

\begin{abstract}
It is shown that the initial value problem (IVP) of the generalized $\mathrm{KdV}$ equation

$$
\partial_{t} u+\partial_{x}(a(u))+\partial_{x}^{3} u=0, \quad u(x, 0)=\phi(x)
$$

is well posed in the classical Sobolev space $H^{s}(R)$ with $s>3 / 4$, which thus establishes a nonlinear map $K$ from $H^{s}(R)$ to $C\left([-T, T] ; H^{s}(R)\right)$. Then it is proved that (i) if $a=a(x)$ is a $C^{\infty}$ function on $R$ to $R$, then $K$ is infinitely many times Frechet differentiable; (ii) if $a=a(x)$ is a polynomial, then $K$ is analytic, i.e. for any $\phi \in H^{s}(R), K$ has a Taylor series expansion
\end{abstract}

$$
K(\phi+h)=\sum_{n=0}^{\infty} \frac{1}{n !} K^{(n)}(\phi)\left[h^{n}\right]
$$

\footnotetext{
*Supported partially by 1992's Taft Summer Research Grant

${ }^{\dagger}$ On leave of absence from: Department of Mathematical Sciences, University of Cincinnati, Ohio 45221
} 
Each term $y_{n}=K^{(n)}\left[h^{n}\right]$ in the series solves a linearized $\mathrm{KdV}$ equation. Thus any "small" perturbation $K(\phi+h)$ of $K(\phi)$ can be obtained by solving a series of linear problems.

The proof of these results relies on various smoothing properties of the associated linear KdV equation.

\section{Introduction}

This paper is mainly concerned with the initial value problem (IVP) for the generalized Korteweg-de Vries (KdV) equation:

$$
\left\{\begin{array}{l}
\partial_{t} u+\partial_{x}(a(u))+\partial_{x}^{3} u=0, \quad t, x \in R \\
u(x, 0)=\phi(x)
\end{array}\right.
$$

in which $a(x)$ is assumed to be a $C^{\infty}$ function on $R$ to $R$, though a weaker differentiablity suffices for most results below.

The KdV equation (i.e., $a(u)=u^{2} / 2$ in (1.1) ) and its generalized form (1.1) have been studied by many authors $[1,2,4-6,10$ - 17, 19 - 23, 27 37, $42-44,47]$. For a complete list of references, see, for example, [ 13, 17, 22,30 ]. In particular, it is well-known that the IVP (1.1) is locally well posed in the classical Sobolev space $H^{s}(R)$ with $s>3 / 2$ (cf. [17]) and is globally well posed with some restrictions on $a(u)$ or the size of the initial data $\phi$ when $s \geq 2$ (see [5], [14], [17], [19] and [28] for the well-posedness of the IVP (1.1) in other function spaces). In the case that $a^{\prime}(u)=u^{k}$ in (1.1) with $k$ being a positive integer, Kenig, Ponce and Vega [23] recently proved that the IVP (1.1) is locally well posed in the space $H^{s}(R)$ with

$$
\begin{cases}s>3 / 4 & \text { if } k=1 \\ s \geq 1 / 4 & \text { if } k=2 \\ s \geq \frac{1}{12} & \text { if } k=3 \\ s \geq \frac{k-4}{2 k} & \text { if } k \geq 4\end{cases}
$$


and is global well-posedness when $1 \leq k \leq 3$ and $s \geq 1$. Their proof is based on careful analysis of various smooth properties of the associated linear problem together with the contraction mapping principle.

In this paper, we first continue to study the well-posedness of the IVP (1.1) in the space $H^{s}(R)$. While only assuming that $a(0)=a^{\prime}(0)=0$, we shall prove that the IVP (1.1) is locally well posed in the space $H^{s}(R)$ with $s>3 / 4$ by using Kenig, Ponce and Vega's argument [22], [23] with a few modification. In order to state our result precisely, we introduce the following Banach spaces as Kenig, Ponce and Vega did in [23].

Let $s>0$ and $T>0$ be given. For

$$
w: \quad R \times[-T, T] \rightarrow R,
$$

define

$$
\begin{gathered}
\lambda_{1}(T, w)=\sup _{[-T, T]}\|w(., t)\|_{s}, \\
\lambda_{2}(T, w)=\left(\sup _{x} \int_{-T}^{T}\left|D^{s} \partial_{x} w(x, t)\right|^{2} d t\right)^{1 / 2}, \\
\lambda_{3}(T, w ; l)=\left(\int_{-T}^{T}\left\|J^{l} \partial_{x} w(., t)\right\|_{\infty}^{4} d t\right)^{1 / 4}
\end{gathered}
$$

with $l \in[0, s-3 / 4]$ where $J^{s}=\left(1-\partial_{x}^{2}\right)^{s / 2}$,

$$
\lambda_{4}(T, w ; r)=(1+T)^{-\rho}\left(\int_{R} \sup _{[-T, T]}\left|J^{r} w(x, t)\right|^{2} d x\right)^{1 / 2}
$$

with $r \in[0, s-3 / 4)$ and $\rho>3 / 4$ being a fixed constant, and

$$
\Lambda_{l, r}^{s}(T ; w)=\max \left\{\lambda_{1}(T, w), \lambda_{2}(T, w), \lambda_{3}(T, w ; l), \lambda_{4}(T, w ; r)\right\} .
$$

Denote by

$$
X_{l, r}^{T, s}=\left\{w \in C\left([-T, T] ; H^{s}(R)\right) \mid \Lambda_{l, r}^{s}(T ; w)<\infty\right\}
$$

with $(l, r) \in\left[0, s-\frac{3}{4}\right] \times\left[0, s-\frac{3}{4}\right)$. This is a Banach space equipped with the norm

$$
\|w\|_{X_{l, r}^{T, s}}:=\Lambda_{l, r}^{s}(T ; w)
$$


Clearly, $X_{l, r}^{T, s}$ is a subspace of $C\left([-T, T] ; H^{s}(R)\right)$ with stronger topology.

We shall prove that if $a(0)=a^{\prime}(0)=0$, then for any $\phi \in H^{s}(R)$, there exists a $T>0$ depending only on $\|\phi\|_{s}$ such that he IVP (1.1) has a unique solution $u \in X_{l, r}^{T, s}$ where $s>3 / 4$ and $(l, r) \in\left[0, s-\frac{3}{4}\right] \times\left[0, s-\frac{3}{4}\right)$. The global result is also obtained while the same restrictions are enforced on $a(u)$ in (1.1) or the size of the initial value $\phi$ as those in Kato [17] but with $s \geq 1$ instead of $s \geq 2$.

Thus the IVP (1.1) establishes a nonlinear map $K$ from $H^{s}(R)$ to $X_{l, r}^{T, s}$ (or $C\left([-T, T] ; H^{s}(R)\right)$ ). In the second part of this paper, we study differentiability of the map $K$. It has been known for many years that the map $K$ is continuous from $H^{s}(R)$ to $C\left([-T, T] ; H^{s}(R)\right)$ [2], [17] and is proved recently by Kenig, Ponce and Vega [23] being Lipschitz continuous in the case that $a^{\prime}(u)=u^{k}$. In our early paper [47], we proved the map $K$ corresponding to the classical $\mathrm{KdV}$ equation, i.e. $a^{\prime}(u)=u$ in (1.1), is infinitely many times Frechet differentiable from $H^{s}(R)$ to $X_{l, r}^{T, s}$ and it has Taylor series expansion at any given $\phi \in H^{s}(R)$. That is to say, the map $K$ is analytic from $H^{s}(R)$ to $X_{l, r}^{T, s}$.

In this paper we shall show that the nonlinear map $K$ established by the IVP (1.1) is also infinitely many times Frechet differentiable from $H^{s}(R)$ to $X_{l, r}^{T, s}$. For any $n \geq 1$, its $n$-th derivative $K^{(n)}(\phi)$ at $\phi \in H^{s}(R)$, a n-linear map from the n-fold product space $\left(H^{s}(R)\right)^{n}$ into $X_{l, r}^{T, s}$, can be constructed by solving a system of inhomogeneous linearized $\mathrm{KdV}$ equations. More precisely, for any $n \geq 1$ and $h_{k} \in H^{s}(R)(k=1,2, \ldots, n)$, denote by

$$
w_{[1, \ldots, n]}^{(n)}:=K^{(n)}(\phi)\left[h_{1}, \ldots, h_{n}\right],
$$

then it solves

$$
\left\{\begin{array}{l}
\partial_{t} w_{[1]}^{(1)}+\partial_{x}\left(a^{\prime}(u) w_{[1]}^{(1)}\right)+\partial_{x}^{3} w_{[1]}^{(1)}=0 \\
w_{[1]}^{(1)}(x, 0)=h(x)
\end{array}\right.
$$

for $n=1$ and

$$
\left\{\begin{array}{l}
\partial_{t} w_{[1, \ldots, n]}^{(n)}+\partial_{x}\left(a^{\prime}(u) w_{[1, \ldots, n]}^{(n)}\right)+\partial_{x}^{3} w_{[1, \ldots, n]}^{(n)}=-\partial_{x}\left(H_{n}\right) \\
w_{[1, \ldots, n]}^{(n)}(x, 0)=0
\end{array}\right.
$$


for $n \geq 2$ where $u=K(\phi)$ and $H_{n}$ is a polynomial of $w_{\left[i_{1}, \ldots, i_{j}\right]}^{(j)}$ with $1 \leq i_{1}, \ldots, i_{j} \leq n$ and $1 \leq j \leq n-1$ (see section 3 for the structure of $H_{n}$ ).

If we choose $h_{1}=h_{2}=\ldots=h$ and denote by

$$
y_{n}=K^{(n)}(\phi)\left[h^{n}\right]
$$

which is a homogeneous polynomial of degree $n$ from $H^{s}(R)$ to $X_{l, r}^{T, s}$, then

$$
\left\{\begin{array}{l}
\partial_{t} y_{1}+\partial_{x}\left(a^{\prime}(u) y_{1}\right)+\partial_{x}^{3} y_{1}=0 \\
y_{1}(x, 0)=h(x)
\end{array}\right.
$$

for $n=1$ and

$$
\left\{\begin{array}{l}
\partial_{t} y_{n}+\partial_{x}\left(a^{\prime}(u) y_{n}\right)+\partial_{x}^{3} y_{n}=-\partial_{x}\left\{M_{n}\right\} \\
y_{n}(x, 0)=0
\end{array}\right.
$$

for $n \geq 2$ where

$$
M_{n}=\sum_{j=2}^{n} \frac{a^{(j)}(u)}{j !} \sum_{k_{1}+\ldots+k_{j}=n} \frac{n !}{k_{1} ! \ldots k_{j} !} y_{k_{1}} \ldots y_{k_{j}}
$$

and the summation $\sum_{k_{1}+\ldots+k_{j}=n}$ is over all possible $\left(k_{1}, \ldots, k_{j}\right)$ with $1 \leq$ $k_{1}, \ldots k_{j} \leq n$ and $k_{1}+\ldots k_{j}=n$.

Thus we can define the n-th Taylor polynomial $P_{n}$ of $K$ at $\phi \in H^{s}(R)$ :

$$
\begin{aligned}
P_{n}(\phi)[h] & :=K(\phi)+\sum_{1}^{n} \frac{K^{(n)}(\phi)}{k !}\left[h^{k}\right] \\
& =u+\sum_{k=1}^{n} \frac{y_{k}}{k !}
\end{aligned}
$$

for any $h \in H^{s}(R)$.

Let

$$
z_{n}=K(\phi+h)-P_{n}(\phi)[h]
$$

It is called the n-th Taylor remainder of $K$ at $\phi$ and we shall see that $z_{n}$ solves

$$
\left\{\begin{array}{l}
\partial_{t} z_{0}+\partial_{x}\left(F_{1}(u, v) z_{0}\right)+\partial_{x}^{3} z_{0}=0 \\
z_{0}(x, 0)=h(x)
\end{array}\right.
$$


for $n=0$ and

$$
\left\{\begin{array}{l}
\partial_{t} z_{n}+\partial_{x}\left(F_{1}(u, v) z_{n}\right)+\partial_{x}^{3} z_{n}=-\partial_{x}\left(G_{n}\right) \\
z_{n}(x, 0)=0
\end{array}\right.
$$

for $n \geq 1$ where

$$
\begin{gathered}
u=K(\phi), \quad v=K(\phi+h), \\
G_{n}=\sum_{m=2}^{n+1} F_{m}(u, v) \sum_{k=0}^{n+1-m} z_{k} \sum_{k_{1}+\ldots+k_{m-1}=n-k} q_{k_{1}} \ldots q_{k_{m-1}}
\end{gathered}
$$

with

$$
q_{m}=\frac{y_{m}}{m !}
$$

and

$$
F_{m}(u, v)=\int_{0}^{1} \lambda_{1}^{m-1} \ldots \int_{0}^{1} \lambda_{m-1} \int_{0}^{1} a^{(m)}\left(\prod_{j=1}^{m} \lambda_{j} v+\left(1-\prod_{j=1}^{m} \lambda_{j}\right) u\right) d \lambda_{m} \ldots d \lambda_{1}
$$

for $m=1, \ldots, n+1$.

Letting $n \rightarrow \infty$ in (1.10), we obtain a formal Taylor series of $K$ at $\phi \in H^{s}(R)$. Assuming that $a(u)$ is a polynomial, we shall prove that for any $\phi \in H^{s}(R)$, there exists a $\delta>0$ such that if $h \in H^{s}(R)$ with $\|h\|_{s} \leq \delta$, then

$$
K(\phi+h)=\sum_{k=0}^{\infty} \frac{K^{(n)}(\phi)}{n !}\left[h^{n}\right]
$$

the series conversing uniformly about $h$ with $\|h\|_{s} \leq \delta$ in the space $X_{l, r}^{T, s}$. In another word, the map $K$ is analytic from $H^{s}(R)$ to $X_{l, r}^{T, s}$.

The paper is organized as follows.

- In section 2, we first list the estimates concerning the IVP

$$
\partial_{t} u+\partial_{x}^{3} u=f(x, t), \quad u(x, 0)=u_{0}(x)
$$

which are needed to establish the nonlinear results. Then we consider the IVP for the following linear equation

$$
\left\{\begin{array}{l}
\partial_{t} u+\partial_{x}(a(v) u)+\partial_{x}^{3} u=f(x, t), \quad x, t \in R \\
u(x, 0)=\phi(x)
\end{array} .\right.
$$


Let $s>3 / 4,(l, r) \in\left[0, s-\frac{3}{4}\right] \times\left[0, s-\frac{3}{4}\right)$ and $T>0$ be given. We shall show that if $v \in X_{0,0}^{T, s}$, then for any $\phi \in H^{s}(R)$ and $f \in L^{1}\left([-T, T] ; H^{s}(R)\right)$, the IVP (1.17) has a unique solution $u \in X_{l, r}^{T, s}$ and

$$
\|u\|_{X_{l, r}^{T, s}} \leq \beta\left(\|v\|_{X_{0,0}^{T, s}}\right)\left(\|\phi\|_{s}+\int_{-T}^{T}\|f(., t)\|_{s} d t\right)
$$

This would be a key estimate to obtain differentiability of the map $K$.

- In section 3, we show that the map $K$ defined by the IVP (1.1) is infinitely many times Frechet differentiable from $H^{s}(R)$ to $X_{l, r}^{T, s}$.

- In section 4, assuming that $a(u)$ in (1.1) is a polynomial, we show that the nonlinear map $K$ is analytic from $H^{s}(R)$ to $X_{l, r}^{T, s}$.

\section{Notations:}

- The norm in $L^{2}(R)$ will be denoted by $\|$.$\| and the norm in H^{s}(R)$ will be denoted by $\|.\|_{s}$. The notation $\|.\|_{\infty}$ is used to denote the norm in $L^{\infty}(R)$.

- $D^{s}=\left(-\partial_{x}^{2}\right)^{s / 2}$ and $J^{s}=\left(1-\partial_{x}^{2}\right)^{s / 2}$ denote the Riesz and the Bessell potential of order $s$ respectively.

- $[A, B]=A B-B A$, where $A, B$ are operators. Thus $\left[J^{s} ; f\right] g=$ $J^{s}(f g)-f J^{s} g$ in which $f$ is regarded as a multiplication operator.

$-H^{\infty}(R):=\cap_{s>0} H^{s}(R)$

- For $1 \leq p, q \leq \infty$ and $f: R \times[-T, T] \rightarrow R$,

$$
\|f\|_{L_{T}^{q} L_{x}^{p}}=\left(\int_{-T}^{T}\left(\int_{-\infty}^{\infty}|f(x, t)|^{p} d x\right)^{\frac{q}{p}} d t\right)^{\frac{1}{q}}
$$

and

$$
\|f\|_{L_{x}^{p} L_{T}^{q}}=\left(\int_{-\infty}^{\infty}\left(\int_{-T}^{T}|f(x, t)|^{q} d t\right)^{\frac{p}{q}} d x\right)^{\frac{1}{p}} .
$$




\section{Linear estimates}

We use $\{W(t)\}_{-\infty}^{+\infty}$ to denote the unitary group which defines the solution of the IVP associated to

$$
\left\{\begin{array}{l}
\partial_{t} v+\partial_{x}^{3} v=0, \quad \text { for } x, t \in R \\
v(x, 0)=v_{0}(x)
\end{array}\right.
$$

where

$$
v(t)=W(t) v_{0}=S_{t} * v_{0}
$$

with $S_{t}($.$) defined by the oscillatory integral$

$$
S_{t}(x)=c \int_{-\infty}^{+\infty} e^{i x \xi} e^{i t \xi^{3}} d \xi
$$

Then the solution of the inhomogeneous equation

$$
\left\{\begin{array}{l}
\partial_{t} v+\partial_{x}^{3} v=f(x, t), \quad x, t \in R \\
v(x, 0)=0
\end{array}\right.
$$

is expressed as

$$
v(t)=\int_{0}^{t} W(t-\tau) f(., \tau) d \tau
$$

Lemma 2.1 For any $s \geq 0$,

$$
\left(\sup _{x} \int_{-\infty}^{\infty}\left|D^{s} \partial_{x} W(t) v_{0}\right|^{2} d t\right)^{1 / 2} \leq c\left\|v_{0}\right\|_{s}
$$

and

$$
\left(\int_{-\infty}^{\infty}\left\|D^{s+1 / 4} W(t) v_{0}\right\|_{\infty}^{4} d t\right)^{1 / 4} \leq c\left\|v_{0}\right\|_{s}
$$

In addition, if $s>3 / 4$, then

$$
\left(\int_{-\infty}^{+\infty} \sup _{[-T, T]}\left|J^{l} W(t) v_{0}\right|^{2}(x) d x\right)^{1 / 2} \leq c(1+T)^{\rho}\left\|v_{0}\right\|_{s}
$$

where $l \in[0, s-3 / 4)$ and $\rho$ is a fixed constant larger than $3 / 4$. 
Proof: see Kenig, Ponce and Vega ([22], Lemma 2.1, Theorem 2.4 and Corollary 2.9).

Remark 2.1 (2.3) is a stronger version of local smoothing effect of Kato type and (2.4) is the global smoothing effect of Strichartz type [38] present in solutions of (2.1). The estimate (2.5), which gives a bound for the associated maximal function $\sup _{[-T, T]}|W(t)$.$| , is due to Vega [46].$

Lemma 2.2 For any $s \geq 0$ and $T>0$,

$$
\left\|W(t) v_{0}\right\|_{s}=\left\|v_{0}\right\|_{s}
$$

and

$$
\sup _{[-T, T]}\left\|\int_{0}^{t} W(t-\tau) f(., \tau) d \tau\right\|_{s} \leq \int_{-T}^{T}\|f(., \tau)\|_{s} d \tau .
$$

Proof: (2.6) and (2.7) follow easily from Kato ([17], Lemma 3.1).

Lemma 2.3 For any $s \geq 0$ and $T>0$,

$$
\left\|D_{x}^{s} \partial_{x} \int_{0}^{t} W(t-\tau) f(., \tau) d \tau\right\|_{L_{x}^{\infty} L_{T}^{2}} \leq c\|f\|_{L^{1}\left([-T, T] ; H^{s}(R)\right)}
$$

and

$$
\left\|D^{s+\frac{1}{4}} \int_{0}^{t} W(t-\tau) f(., \tau) d \tau\right\|_{L_{x}^{\infty} L_{T}^{4}} \leq c \int_{-T}^{T}\|f(., \tau)\|_{s} d \tau .
$$

If $s>3 / 4$, then

$$
\left\|J^{l} \int_{0}^{t} W(t-\tau) f(., \tau) d \tau\right\|_{L_{x}^{2} L_{T}^{\infty}} \leq c(1+T)^{\rho} \int_{-T}^{T}\|f(., \tau)\|_{s} d \tau
$$

where $l \in[0, s-3 / 4)$ and $\rho$ is a fixed constant larger than $3 / 4$.

Proof: It follows from Lemma 2.1 by using Minkowski's integral inequality (see $[47])$.

Lemma 2.4 Let $s>1 / 2$ and $T>0$ be given. Then there is a constant $c>0$ such that

$$
\int_{-T}^{T}\left\|u \partial_{x} v\right\|_{s} d t \leq c T^{1 / 2}(1+T)^{\rho}\|u\|_{X_{0,0}^{T, s}}\|v\|_{X_{0,0}^{T, s}}
$$


and

$$
\int_{-T}^{T}\left\|\partial_{x}(u v)\right\|_{s} d t \leq c T^{1 / 2}(1+T)^{\rho}\|u\|_{X_{0,0}^{T, s}}\|v\|_{X_{0,0}^{T, s}}
$$

for any $u, v \in X_{0,0}^{T, s}$.

Proof: see Lemma 2.4 in [47].

Lemma 2.5 Let $s>0$ be given. Then there is a constant $c>0$ such that for any $y_{k} \in H^{s}(R), k=1,2, \ldots, m$,

$$
\left\|\prod_{k=1}^{m} y_{k}\right\|_{s} \leq c^{m}\left(\sum_{j=1}^{m}\left\|y_{j}\right\|_{s} \prod_{k=1, k \neq j}^{m}\left\|y_{k}\right\|_{\infty}\right)
$$

and if $s>1 / 2$,

$$
\left\|\prod_{k=1}^{m} y_{k}\right\|_{s} \leq c^{m} \prod_{k=1}^{m}\left\|y_{k}\right\|_{s}
$$

for any $m \geq 2$ where $c$ in (2.14) may be different from $c$ in (2.13).

Proof: According to Kato and Ponce ([18], Lemma X4),

$$
\left\|y_{1} y_{2}\right\|_{s} \leq c\left\{\left\|y_{1}\right\|_{s}\left\|y_{2}\right\|_{\infty}+\left\|y_{2}\right\| s\left\|y_{1}\right\|_{\infty}\right\}
$$

which is (2.13) with $m=2$. Suppose that (2.13) is true for $m=N$. Then for $m=N+1$,

$$
\begin{aligned}
\left\|\prod_{k=1}^{N+1} y_{k}\right\|_{s} & =\left\|y_{N+1} \prod_{k=1}^{N} y_{k}\right\|_{s} \\
& \leq c\left\{\left\|y_{N+1}\right\|_{s}\left\|\prod_{k=1}^{N} y_{k}\right\|_{\infty}+\left\|y_{N+1}\right\|_{\infty}\left\|\prod_{k=1}^{N} y_{k}\right\|_{s}\right\} \\
& \leq c\left\|y_{N+1}\right\|_{s} \prod_{k=1}^{N}\left\|y_{k}\right\|_{\infty}+c^{N+1} \sum_{j=1}^{N}\left\|y_{j}\right\|_{s} \prod_{k=1, k \neq j}^{N+1}\left\|y_{k}\right\|_{\infty} \\
& \leq c^{N+1} \sum_{j=1}^{N+1}\left\|y_{j}\right\|_{s} \prod_{k=1, k \neq j}^{N+1}\left\|y_{k}\right\|_{\infty}
\end{aligned}
$$

Thus (2.13) is proved by induction. As for (2.14), it follows directly from (2.13) since $\left\|y_{k}\right\|_{\infty} \leq\left\|y_{k}\right\|_{s}$ if $s>1 / 2$. The proof is completed. $\square$ 
Lemma 2.6 Let $b \in C^{\infty}(R ; R)$ with $b(0)=0$. Then

$$
\|b(u)\|_{s} \leq \tilde{b}\left(\|u\|_{s}\right), \quad s>1 / 2
$$

where $\tilde{b}($.$) is a monotone increasing function depending only on b$.

Proof: see Kato ([17], Lemma A.3).

Lemma 2.7 Let $s>1 / 2$ and $T>0$ be given and assume $a \in C^{\infty}(R ; R)$ with $a(0)=0$. Then there is a constant $c>0$ such that for any $u \in X_{0,0}^{T, s}$ and $y \in X_{0,0}^{T, s}$,

$$
\int_{-T}^{T}\left\|\partial_{x}(a(u) y)\right\|_{s} d t \leq c \beta\left(\|u\|_{X_{0,0}^{T, s}}\right)\|y\|_{X_{0,0}^{T, s}}
$$

where $\beta($.$) is a continuous monotone increasing function only depending on$ $a$.

Proof: First of all,

$$
\left\|\partial_{x}(a(u) y)\right\|_{s} \leq\left\|a^{\prime}(u) y \partial_{x} u\right\|_{s}+\left\|a(u) \partial_{x} y\right\|_{s}
$$

and it is easy to see by using Lemma 2.5 and Lemma 2.6 that

$$
\begin{aligned}
& \left\|a^{\prime}(u) y \partial_{x} u\right\|_{s} \leq\left\|\left(a^{\prime}(u)-a^{\prime}(0)\right) y \partial_{x} u\right\|_{s}+\left|a^{\prime}(0)\right|\left\|y \partial_{x} u\right\|_{s} \\
& \leq c\left\{\left\|a^{\prime}(u)-a^{\prime}(0)\right\|_{s}\left\|y \partial_{x} u\right\|_{\infty}+\left\|a^{\prime}(u)-a^{\prime}(0)\right\|_{\infty}\left\|y \partial_{x} u\right\|_{s}\right\}+ \\
& \quad+\left|a^{\prime}(0)\right|\left\|y \partial_{x} u\right\|_{s} \\
& \leq c\left\{\left\|a^{\prime}(u)-a^{\prime}(0)\right\|_{s}+\left|a^{\prime}(0)\right|\right\}\left\|y \partial_{x} u\right\|_{s} \\
& \leq c \beta_{1}\left(\|u\|_{s}\right)\left\|y \partial_{x} u\right\|_{s}
\end{aligned}
$$

where $\beta_{1}():. R^{+} \rightarrow R^{+}$is a continuous monotone increasing function only depending on $a$. Using Lemma 2.4 yields

$$
\begin{aligned}
\int_{-T}^{T}\left\|a^{\prime}(u) y \partial_{x} u\right\|_{s} d t & \leq c \sup _{[-T, T]} \beta\left(\|u\|_{s}\right) \int_{-T}^{T}\left\|y \partial_{x} u\right\|_{s} d t \\
& \leq c T^{1 / 2}(1+T)^{\rho} \beta_{1}\left(\|u\|_{X_{0,0}^{T, s}}\right)\|u\|_{X_{0,0}^{T, s}}\|y\|_{X_{0,0}^{T, s}}
\end{aligned}
$$


In addition, applying Lemma 2.10 in [22], we obtain

$$
\begin{aligned}
& \left\|a(u) \partial_{x} y\right\|_{s}=\left\|J^{s}\left(a(u) \partial_{x} y\right)\right\| \\
& =\left\|a(u) D^{s} \partial_{x} y+a(u)\left(J^{s}-D^{s}\right) \partial_{x} y+\left[J^{s} ; a(u)\right] \partial_{x} y\right\| \\
& \leq\left\|a(u) D^{s} \partial_{x} y\right\|+\|a(u)\|_{\infty}\|y\|_{s}+c\left\{\left\|\partial_{x} y\right\|_{\infty}\|a(u)\|_{s}+\right. \\
& \left.\quad+\left\|a^{\prime}(u) \partial_{x} u\right\|_{\infty}\|y\|_{s}\right\} .
\end{aligned}
$$

Note that

$$
\begin{aligned}
\int_{-T}^{T}\|a(u)\|_{\infty}\|y\|_{s} d t & \leq \sup _{[-T, T]}\|a(u)\|_{s} \int_{-T}^{T}\|y\|_{s} d t \\
& \leq c T \sup _{[-T, T]} \beta_{2}\left(\|u\|_{s}\right) \sup _{[-T, T]}\|y\|_{s} \quad \text { (by Lemma 2.6) } \\
& \leq c T^{1 / 2}(1+T)^{\rho} \beta_{2}\left(\|u\|_{X_{0,0}^{T, s}}\right)\|y\|_{X_{0,0}^{T, s}}
\end{aligned}
$$

where $\beta_{2}():. R^{+} \rightarrow R^{+}$is a continuous monotone increasing function depending only on $a$,

$$
\begin{aligned}
\int_{-T}^{T}\|a(u)\|_{s}\left\|\partial_{x} y\right\|_{\infty} d t & \leq \sup _{[-T, T]}\|a(u)\|_{s} \int_{-T}^{T}\left\|\partial_{x} y\right\|_{\infty} d t \\
& \leq \sup _{[-T, T]} \beta_{2}\left(\|u\|_{s}\right) \int_{-T}^{T}\left\|\partial_{x} y\right\|_{\infty} d t \\
& \leq \beta_{2}\left(\|u\|_{X_{0,0}^{T, s}}\right)(2 T)^{3 / 4}\left(\int_{-T}^{T}\left\|\partial_{x} y\right\|_{\infty}^{4} d t\right)^{1 / 4} \\
& \leq c T^{1 / 2}(1+T)^{\rho} \beta_{2}\left(\|u\|_{X_{0,0}^{T, s}}\right)\|y\|_{X_{0,0}^{T, s}}, \\
\int_{-T}^{T}\left\|a^{\prime}(u) \partial_{x} u\right\|_{\infty}\|y\|_{s} d t & \leq \sup _{[-T, T]}\left(\left\|a^{\prime}(u)\right\|_{\infty}\|y\|_{s}\right) \int_{-T}^{T}\left\|\partial_{x} u\right\|_{\infty} d t \\
& \leq c T^{1 / 2}(1+T)^{\rho} \beta_{3}\left(\|u\|_{X_{0,0}^{T, s}}\|u\|_{X_{0,0}^{T, s}}\|y\|_{X_{0,0}^{T, s}},\right.
\end{aligned}
$$

where

$$
\beta_{3}(r)=\sup _{|\lambda| \leq r}\left|a^{\prime}(\lambda)\right|,
$$

and

$$
\int_{-T}^{T}\left\|a(u) D^{s} \partial_{x} y\right\| d t \leq T^{1 / 2} \int_{-T}^{T} \int_{R}\left|a(u) D^{s} \partial_{x} u\right|^{2} d x d t
$$




$$
\begin{aligned}
& \leq \int_{R} \int_{-T}^{T}|u|^{2}\left|D^{s} \partial_{x} u\right|^{2} d x d t \sup _{[-T, T]}\left\|\frac{a(u)}{u}\right\|_{\infty} \\
& \leq T^{1 / 2} \beta_{4}\left(\|u\|_{X_{0,0}^{T, s}}\right)\left(\int_{R} \sup _{[-T, T]}|u(x, t)|^{2} d x\right)^{1 / 2}\left(\sup _{x} \int_{-T}^{T}\left|D^{s} \partial_{x} y\right|^{2} d t\right)^{1 / 2} \\
& \leq c T^{1 / 2}(1+T)^{\rho} \beta_{4}\left(\|u\|_{X_{0,0}^{T, s}}\|u\|_{X_{0,0}^{T, s}}\|y\|_{X_{0,0}^{T, s}}\right.
\end{aligned}
$$

where

$$
\beta_{4}(r)=\sup _{|\lambda| \leq r}\left|\frac{a(\lambda)}{\lambda}\right|
$$

Therefore,

$$
\int_{-T}^{T}\left\|\partial_{x}(a(u) y)\right\|_{s} d t \leq c T^{1 / 2}(1+T)^{\rho} \beta\left(\|u\|_{X_{0,0}^{T, s}}\right)\|y\|_{X_{0,0}^{T, s}}
$$

for some constant $c>0$ where

$$
\beta(r)=\max \left\{r \beta_{1}(r), \beta_{2}(r), r \beta_{3}(r), r \beta_{4}(r)\right\} .
$$

The proof is completed.

¿From the proof of the above lemma we may draw the following corollary.

Corollary 2.1 Let $s>1 / 2$ and $T>0$ be given and assume $a \in C^{\infty}(R ; R)$ with $a^{\prime}(0)=0$. Then for any $u \in X_{0,0}^{T, s}$,

$$
\int_{-T}^{T}\left\|\partial_{x}(a(u))\right\|_{s} d t \leq c \beta\left(\|u\|_{X_{0,0}^{T, s}}\right) T^{1 / 2}(1+T)^{\rho}\|u\|_{X_{0,0}^{T, s}}
$$

where $\beta($.$) is a continuous monotone increasing function only depending on$ a.

Lemma 2.8 Let $s>1 / 2, T>0$ and $a \in C^{\infty}(R ; R)$ be given. Then there exists a constant $c>0$ such that for any $u \in X_{0,0}^{T, s}$ and $y_{k} \in X_{0,0}^{T, s}$ with $k=1,2, \ldots, m$ and $m \geq 2$,

$$
\int_{-T}^{T}\left\|\partial_{x}\left(a(u) \prod_{k=1}^{m} y_{k}\right)\right\|_{s} d t \leq c^{m} \beta\left(\|u\|_{X_{0,0}^{T, s}}\right) \prod_{k=1}^{m}\left\|y_{k}\right\|_{X_{0,0}^{T, s}}
$$

where $\beta($.$) is a continuous monotone increasing function only depending on$ a. 
Proof: By applying (2.14) we have

$$
\begin{aligned}
& \left\|\partial_{x}\left(a(u) \prod_{k=1}^{m} y_{k}\right)\right\|_{s} \leq\left\|a^{\prime}(u) \partial_{x} u \prod_{k=1}^{m} y_{k}\right\|_{s}+\sum_{k=1}^{m}\left\|\prod_{j=1, j \neq k}^{m} a(u) y_{j} \partial_{x} y_{k}\right\|_{s} \\
& \leq c^{m+1}\left(\left\|a^{\prime}(u)-a^{\prime}(0)\right\|_{s}+\left|a^{\prime}(0)\right|\right)\left\|y_{1} \partial_{x} u\right\|_{s} \prod_{k=2}^{m}\left\|y_{k}\right\|_{s}+ \\
& +c^{m+1}\left(\|a(u)-a(0)\|_{s}+|a(0)|\right)\left(\sum_{k=2}^{m}\left\|y_{1} \partial_{x} y_{k}\right\|_{s} \prod_{j=2, j \neq k}^{m}\left\|y_{j}\right\|_{s}+\right. \\
& \left.\quad+\left\|y_{2} \partial_{x} y_{1}\right\|_{s} \prod_{j=3}^{m}\left\|y_{j}\right\|_{s}\right) .
\end{aligned}
$$

Thus, using Lemma 2.6 and Lemma 2,4, we have

$$
\begin{aligned}
& \int_{-T}^{T}\left\|\partial_{x}\left(a(u) \prod_{k=1}^{m} y_{k}\right)\right\|_{s} d t \leq c_{1}^{m+1} \sup _{[-T, T]}\left(\left\|a^{\prime}(u)-a^{\prime}(0)\right\|_{s}+\left|a^{\prime}(0)\right|\right) * \\
& * \prod_{k=2[-T, T]}^{m} \sup _{[-T}\left\|y_{k}\right\|_{s} \int_{-T}^{T}\left\|y_{1} \partial_{x} u\right\|_{s} d t+c_{1}^{m+1} \sup _{[-T, T]}\left(\|a(u)-a(0)\|_{s}+|a(0)|\right) * \\
& *\left(\sum_{k=2}^{m} \sup _{-T, T]} \prod_{j=2, j \neq k}^{m}\left\|y_{j}\right\|_{s} \int_{-T}^{T}\left\|y_{1} \partial_{x} y_{k}\right\|_{s} d t+\prod_{j=3}^{m} \sup _{[-T, T]}\left\|y_{j}\right\|_{s} \sup _{[-T, T]}\left\|y_{2} \partial_{x} y_{1}\right\|_{s}\right) \\
& \leq c_{1}^{m+1} \beta_{1}\left(\|u\|_{X_{0,0}^{T, s}}\right) T^{1 / 2}(1+T)^{\rho}\|u\|_{X_{0,0}^{T, s}} \prod_{k=1}^{m}\left\|y_{k}\right\|_{X_{0,0}^{T, s}}+ \\
& \quad+m c_{1}^{m+1} \beta_{1}\left(\|u\|_{X_{0,0}^{T, s}}\right) T^{1 / 2}(1+T)^{\rho} \prod_{k=1}^{m}\left\|y_{k}\right\|_{X_{0,0}^{T, s}} \\
& \leq c^{m} \beta\left(\|u\|_{\left.X_{0,0}^{T, s}\right)} \prod_{k=1}^{m}\left\|y_{k}\right\|_{X_{0,0}^{T, s}}\right.
\end{aligned}
$$

for some $c>0$. The proof is completd.

To end this section, we consider the following linear problem:

$$
\left\{\begin{array}{l}
\partial_{t} u+\partial_{x}(a(v) u)+\partial_{x}^{3} u=f(x, t), \quad x, t \in R \\
u(x, 0)=\phi(x)
\end{array}\right.
$$

where $a(.) \in C^{\infty}(R ; R)$ with $a(0)=0$. 
Theorem 2.1 Let $s>3 / 4,(l, r) \in\left[0, s-\frac{3}{4}\right] \times\left[0, s-\frac{3}{4}\right), T>0$ and $v \in X_{0,0}^{T, s}$ be given. Then for any $f \in L^{1}\left([-T, T] ; H^{s}(R)\right)$ and $\phi \in H^{s}(R)$, there exists a unique solution $u \in X_{l, r}^{T, s}$ to (D.18) such that

$$
\|u\|_{X_{l, r}^{T, s}} \leq \beta\left(\|v\|_{X_{0,0}^{T, s}}\right)\left(\|\phi\|_{s}+\int_{-T}^{T}\|f(., t)\|_{s} d t\right)
$$

where $\beta$ is a continuous monotone increasing function only depending on a.

Proof: We use the contraction principle argument that Kenig, Ponce and Vega used in [23].

For any given $\phi \in H^{s}(R)$ and $f \in L^{1}\left([-T, T] ; H^{s}(R)\right)$, denote by $u=$ $\Phi(w)$, the solution of the following IVP

$$
\left\{\begin{array}{l}
\partial_{t} u+\partial_{x}^{3} u=f-\partial_{x}(a(v) w) \\
u(x, 0)=\phi(x)
\end{array}\right.
$$

where

$$
w \in S_{b}^{T}=\left\{w \in X_{0,0}^{T, s} \mid \quad \Lambda_{0,0}^{s}(T ; w) \leq b\right\}
$$

for some $b>0$ to be determined.

We shall show that there exists a $b=b\left(\|\phi\|_{s},\|f\|_{L^{1}\left([-T, T] ; H^{s}(R)\right)}\right)>0$ and a $T^{*}>0$ such that $u=\Phi(w) \in S_{b}^{T^{*}}$ if $w \in S_{b}^{T^{*}}$ and

$$
\Phi: S_{b}^{T^{*}} \rightarrow S_{b}^{T^{*}}
$$

is a contraction map.

Consider the integral equation form of the IVP (2.20),

$$
u(t)=W(t) \phi+\int_{0}^{t} W(t-\tau) \partial_{x}(a(v) w)(., \tau) d \tau .
$$

Applying (2.3) - (2.10) and (2.15) to (2.21) leads to

$$
\begin{aligned}
& \Lambda_{l, r}^{s}(t ; u) \leq c\left(\|\phi\|_{s}+\int_{-t}^{t}\|f(., \tau)\|_{s} d \tau\right)+c \int_{-t}^{t}\left\|\partial_{x}(a(v) w)\right\|_{s} d \tau \\
& \leq c\left(\|\phi\|_{s}+\int_{-T}^{T}\|f(., \tau)\|_{s} d \tau\right)+ \\
& \quad c t^{1 / 2}(1+t)^{\rho} \beta\left(\|v\|_{X_{0,0}^{T, s}}\right) \Lambda_{0,0}^{s}(t ; w)
\end{aligned}
$$


for $(l, r) \in\left[0, s-\frac{3}{4}\right] \times\left[0, s-\frac{3}{4}\right)$. In particular,

$$
\Lambda_{0,0}^{s}(t ; u) \leq c\left(\|\phi\|_{s}+\int_{-T}^{T}\|f\|_{s} d \tau\right)+c \beta\left(\|v\|_{X_{0,0}^{T, s}}\right) t^{1 / 2}(1+t)^{\rho} \Lambda_{0,0}^{s}(t ; w) .
$$

Choosing

$$
b=2\left(\|\phi\|_{s}+\int_{-T}^{T}\|f\|_{s} d \tau\right)
$$

and $0<T^{*}<T$ such that

$$
c T^{*}\left(1+T^{*}\right)^{\rho} \beta\left(\|v\|_{X_{0,0}^{T, s}}\right)=\frac{1}{2},
$$

we obtain

$$
\Lambda_{0,0}^{s}\left(T^{*} ; u\right) \leq b .
$$

Thus, $\Phi$ is a map from $S_{b}^{T^{*}}$ to $S_{b}^{T^{*}}$.

For any $w_{1}, w_{2} \in S_{b}^{T^{*}}$, let

$$
z=\Phi\left(w_{1}\right)-\Phi\left(w_{2}\right)
$$

Then

$$
z(t)=\int_{0}^{t} W(t-\tau) \partial_{x}\left(a(v)\left(w_{1}-w_{2}\right)\right) d \tau
$$

and

$$
\begin{aligned}
\Lambda_{0,0}^{s}\left(T^{*} ; z\right) & \leq c \int_{-T^{*}}^{T^{*}}\left\|\partial_{x}\left(a(v)\left(w_{1}-w_{2}\right)\right)\right\|_{s} d \tau \\
& \leq c \beta\left(\|v\|_{\left.X_{0,0}^{T, s}\right)} \sqrt{T^{*}}\left(1+T^{*}\right)^{\rho} \Lambda_{0,0}^{s}\left(T^{*} ; w_{1}-w_{2}\right)\right.
\end{aligned}
$$

It follows from (2.24) that

$$
\Lambda_{0,0}^{s}\left(T^{*} ; z\right) \leq \frac{1}{2} \Lambda_{0,0}^{s}\left(T^{*}, w_{1}-w_{2}\right) .
$$

Consequently, by the contraction principle, there exists a unique solution $u \in S_{b}^{T^{*}}$ such that

$$
\Phi(u)=u
$$

i.e.

$$
u(t)=W(t) \phi-\int_{0}^{t} W(t-\tau)\left(\partial_{x}(a(v) u)\right)(\tau) d \tau+\int_{0}^{t} W(t-\tau) f(., \tau) d \tau
$$


for $-T^{*}<t<T^{*}$. In addition, it follows from (2.22), (2.23) and (2.25) that

$$
\Lambda_{l, r}^{s}\left(T^{*} ; u\right) \leq c\left(\|\phi\|_{s}+\int_{-T}^{T}\|f\|_{s} d \tau\right)
$$

for $(l, r) \in\left[0, s-\frac{3}{4}\right] \times\left[0, s-\frac{3}{4}\right)$.

Finally, note that $T^{*}$ determined by (2.24) only depends on $\beta\left(\|v\|_{X_{0,0}^{T, s}}\right)$ and, in particular, it does not depend on $f$ and $\phi$. Thus, a standard argument shows that $T^{*}$ can be extended to $T^{*}=T$ and (2.28) hold for $T^{*}=T$ with another $c$ depending only on $\|v\|_{X_{0,0}^{T, s}}$ and $a$. The proof is completed.

Remark 2.2 Theorem 2.1 is still true if $a(v)$ in (D.18) is replaced by

$$
\int_{0}^{1} a\left(\lambda v_{1}+(1-\lambda) v_{2}\right) d \lambda
$$

with $v_{1}, v_{2} \in X_{0,0}^{T, s}$.

\section{Well-posedness}

In this section we consider the IVP of the generalized KdV equation

$$
\left\{\begin{array}{l}
\partial_{t} u+\partial_{x}(a(u))+\partial_{x}^{3} u=0, \quad x, t \in R \\
u(x, 0)=\phi(x)
\end{array}\right.
$$

where $a=a(x)$ is assumed to be a $C^{\infty}$ function from $R$ to $R$ and

$$
a(0)=a^{\prime}(0)=0 .
$$

The following result is due to Kato [17]

Proposition 3.1 Let $s>3 / 2$ be given. Then

(i) For each $\phi \in H^{s}(R)$, there exists a $T>0$ depending only on $\|\phi\|_{s}$, and a unique solution $u$ to (3.1) such that

$$
u \in C\left([-T, T] ; H^{s}(R)\right) .
$$


The map $\phi \rightarrow u$ is continuous from $H^{s}(R)$ to $C\left([-T, T] ; H^{s}(R)\right)$.

(ii). If, in addition, $\phi \in H^{s^{\prime}}(R)$ for some $s^{\prime}>s$, then (i) is true with same $T$ and with s replaced by $s^{\prime}$.

(iii). There is a number $\gamma_{a}>0$ depending only on a, which is called the ceiling for the given a, such that if $\phi \in H^{s}(R)$ with $s \geq 2$ and

$$
\|\phi\|_{1} \leq \gamma_{a}
$$

then there is a unique solution $u$ to (3.1) such that

$$
u \in C\left([0, \infty) ; H^{s}(R)\right) .
$$

Based on Kato's results, we shall prove the following thoerems by using Kenig, Ponce and Vega's argument in [22] with a few modification.

Theorem 3.1 Let $s>3 / 4$ be given. Then

(i). For any $\phi \in H^{s}(R)$, there exists a $T>0$ depending only on $\|\phi\|_{s}$, and a unique solution $u \in C\left([-T, T] ; H^{s}(R)\right)$ to the IVP (1.1) satisfying

$$
\left(\int_{-T}^{T}\left\|\partial_{x} u(., t)\right\|_{\infty}^{4} d t\right)^{1 / 4}<\infty
$$

and moreover,

$$
\|u\|_{X_{l, r}^{T, s}} \leq \eta\left(\|\phi\|_{s}\right)
$$

where $(l, r) \in\left[0, s-\frac{3}{4}\right] \times\left[0, s-\frac{3}{4}\right)$ and $\eta($.$) is a continuous monotone$ increasing function with $\eta(0)=0$.

(ii). For any $T^{\prime}<T$, there exists a neighborhood $U$ of $\phi$ in $H^{s}(R)$ such that the map

$$
K: \phi \rightarrow u(., t)
$$

from $U$ to $X_{l, r}^{T, s}$ is Lipschitz continuous.

Theorem 3.2 Let $s \geq 1$ be given. Then Theorem 1.1 is true with $T$ arbitrarily large provided that

$$
\|\phi\|_{1} \leq \gamma_{a}
$$

where $\gamma_{a}$ is the ceiling of a defined in Theorem 3.1. 
Remark 3.1 According to Kato [17]

$$
\gamma_{a}=\infty
$$

if

$$
\lim _{|\lambda| \rightarrow \infty} \sup |\lambda|^{-6} a_{2}(\lambda)=0
$$

where

$$
a_{2}(\lambda)=2 \int_{0}^{\lambda}(\lambda-\mu) a^{\prime}(\mu) d \mu .
$$

Note that (3.3) is true if

$$
\lim _{|\lambda| \rightarrow \infty} \sup |\lambda|^{-4} a^{\prime}(\lambda)=0 .
$$

or

$$
a^{\prime}(u) \leq|u|^{p}
$$

with $p \leq 3$ as $|u| \rightarrow \infty$.

Remark 3.2 Theorem 3.2 follows from Theorem 3.1 and the global a priori estimates for solutions of the IVP (3.1) due to Kato [17] by a standard argument.

We start to prove Theorem 3.1 by establishing the following a priori estimate for solutions of (3.1).

Proposition 3.2 Let $s>3 / 4, T>0$ and $(l, r) \in\left[0, s-\frac{3}{4}\right] \times\left[0, s-\frac{3}{4}\right)$ be given. If $u \in X_{0,0}^{T, s}$ is a solution of (3.1), then there exists a $T_{0}>0$ depending only on $\|\phi\|_{s}$ such that

$$
\Lambda_{l, r}^{s}\left(T_{0} ; u\right)<c\|\phi\|_{s}
$$

where $c>0$ is a constant independent of $u$.

Proof: Consider the integral equation form of (3.1),

$$
u(t)=W(t) \phi-\int_{0}^{t} W(t-\tau) \partial_{x}(a(u))(\tau) d \tau
$$


Applying (2.3) - (2.10) to (3.1) yields

$$
\Lambda_{l, r}^{s}(t ; u) \leq c\|\phi\|_{s}+c \int_{-t}^{t}\left\|\partial_{x}(a(u))(\tau)\right\|_{s} d \tau
$$

for any $t \leq T$. In particular, using (2.16), we have

$$
\begin{aligned}
\Lambda_{0,0}^{s}(t ; u) & \leq c\|\phi\|_{s}+c \int_{-t}^{t}\left\|\partial_{x}(a(u))(\tau)\right\|_{s} d \tau \\
& \leq c\|\phi\|_{s}+c \beta\left(\Lambda_{0,0}^{s}(t ; u)\right) t^{1 / 2}(1+t)^{\rho} \Lambda_{0,0}^{s}(t ; u) .
\end{aligned}
$$

Note that $\beta\left(\Lambda_{0,0}^{s}(t ; u)\right)$ is a continuous increasing function of $t$. There exists a $t=T_{0}$ such that

$$
c \beta\left(\Lambda_{0,0}^{s}\left(T_{0} ; u\right)\right) T_{0}^{1 / 2}\left(1+T_{0}\right)^{\rho}=1 / 2
$$

and it follows from (3.7) that

$$
\Lambda_{0,0}^{s}\left(T_{0} ; u\right) \leq 2 c\|\phi\|_{s}
$$

Thus the following inequality must hold

$$
c \beta\left(2 c\|\phi\|_{s}\right) T_{0}^{1 / 2}\left(1+T_{0}\right)^{\rho} \geq 1 / 2,
$$

which implies that $T_{0}>M_{1}>0$ with $M_{1}>0$ depending only on $\|\phi\|_{s}$. (3.4) follows from (3.6), (2.16) and (3.9). The proof is completed.

Proposition 3.3 Let $s>3 / 4$ and $(l, r) \in\left[0, s-\frac{3}{4}\right] \times\left[0, s-\frac{3}{4}\right)$ be given . For any $\phi \in H^{s}(R)$, suppose $\phi_{\epsilon} \in H^{\infty}, \epsilon \in(0,1)$, with

$$
\lim _{\epsilon \rightarrow 0} \phi_{\epsilon}=\phi_{0} \quad \text { in } H^{s}(R) .
$$

Then there exist $T>0$ such that for any $\epsilon \in(0,1)$, (3.1) has a unique solution $u_{\epsilon} \in C\left([-T, T] ; H^{\infty}(R)\right)$ with $u_{\epsilon}(x, 0)=\phi_{\epsilon}(x)$ satisfying

$$
\Lambda_{l, r}^{s}\left(T ; u_{\epsilon}\right)<c\left\|\phi_{\epsilon}\right\|_{s}
$$

for $0<\epsilon<1$ where $c$ is the same constant as that in (3.4). 
Proof: According to Theorem 3.1, $u_{\epsilon} \in C\left(\left[-T_{\epsilon}, T_{\epsilon}\right] ; H^{\infty}(R)\right)$ where $T_{\epsilon}$ only depends on $\left\|\phi_{\epsilon}\right\|_{s}$. Since $\left\|\phi_{\epsilon}\right\|_{s}$ is uniformly bounded for $\epsilon \in(0,1)$, we may assume that

$$
T_{\epsilon}>T_{1} \quad \text { for any } \epsilon \in(0,1)
$$

for some $T_{1}>0$ and therefore

$$
u_{\epsilon} \in X_{0.0}^{T_{1}, s} .
$$

Thus it follows from Proposition 3.2 that

$$
\Lambda_{l, r}^{s}\left(T ; u_{\epsilon}\right) \leq c\left\|\phi_{\epsilon}\right\|_{s}
$$

where $T>0$ is a constant independent of $\epsilon$ since $\left\|\phi_{\epsilon}\right\|_{s}$ is bounded. The proof is completed.

Proof of Theorem 3.1 For $\phi \in H^{s}(R)$, choose $\phi_{\epsilon} \in H^{\infty}(R)$ such that

$$
\lim _{\epsilon \rightarrow 0} \phi_{\epsilon}=\phi \quad \text { in } H^{s}(R) .
$$

By Proposition 3.3, there exists a $T>0$ and $K>0$ such that for any $\epsilon \in(0,1)$ the IVP

$$
\left\{\begin{array}{l}
\partial_{t} u_{\epsilon}+\partial_{x}\left(a\left(u_{\epsilon}\right)\right)+\partial_{x}^{3} u_{\epsilon}=0 \\
u_{\epsilon}(x, 0)=\phi_{\epsilon}(x)
\end{array}\right.
$$

has a unique solution $u_{\epsilon} \in C\left([-T, T] ; H^{\infty}(R)\right)$ satisfying

$$
\Lambda_{l, r}^{s}\left(T ; u_{\epsilon}\right)<K .
$$

It suffices to show that $u_{\epsilon}$ is a Cauchy sequence in $X_{l, r}^{T, s}$ and then the limit $u$ of $u_{\epsilon}$ as $\epsilon \rightarrow 0$ is the desired solution of the IVP (3.1) corresponding to the initial value $\phi$.

Let $\epsilon^{\prime}<\epsilon$ and

$$
w=u_{\epsilon}-u_{\epsilon^{\prime}} .
$$

Then $w$ solves

$$
\left\{\begin{array}{l}
\partial_{t} w+\partial_{x}\left(A\left(u_{\epsilon}, u_{\epsilon^{\prime}}\right) w\right)+\partial_{x}^{3} w=0 \\
w(x, 0)=\phi_{\epsilon}-\phi_{\epsilon^{\prime}}
\end{array}\right.
$$


where

$$
A(u, v)=\int_{0}^{1} a^{\prime}(\lambda u-(1-\lambda)(v)) d \lambda
$$

According to Theorem 2.1 and its remark,

$$
\Lambda_{l, r}^{s}(T ; w) \leq c^{*}\left\|\phi_{\epsilon}-\phi_{\epsilon^{\prime}}\right\|_{s}
$$

for some $c^{*}>0$ independent of $\epsilon$ and therefore $u_{\epsilon}$ is a Cauchy sequence in $X_{l, r}^{T, s}$.

Finally, if $T^{\prime}<T$, (3.1) defines a map $k$ from a neighborhood $U$ of $\phi$ in $H^{s}(R)$ to $X_{l, r}^{T, s}$. For any $\psi \in H^{s}(R)$ with $\|\psi-\phi\|_{s} \leq \epsilon_{0}$ for some $\epsilon_{0}>0$, let $u$ and $v$ be the solutions of the IVP (3.1) with $u(x, 0)=\phi(x)$ and $v(x, 0)=\psi(x)$, respectively. Then, similarly, we have

$$
\Lambda_{l, r}^{s}\left(T^{\prime} ; u-v\right) \leq c\|\phi-\psi\|_{s}
$$

where $c$ depends only on $\|\phi\|_{s}$. Therefore the map $\phi \rightarrow u$ is Lipschitz continuous. The proof is completed.

\section{Diffenrentiability}

Let $X, Y$ be two Banach spaces. An $n$-linear map from $X$ to $Y$ is a map from the $n$-fold product space $X^{n}$ into $Y$ such that

$$
x_{k} \rightarrow f\left(x_{1}, \ldots, x_{n}\right)
$$

is linear for each fixed $\left(x_{1}, x_{2}, \ldots, x_{k-1}, x_{k+1}, \ldots, x_{n}\right)$, for $k=1,2, \ldots, n$. A homogeneous polynomial of degree $n$ from $X$ to $Y$ is a map of the form $x \rightarrow f(x, \ldots, x)$, for some $n$-linea map $f$.

A map $f: X \rightarrow Y$ is Frechet differentiable at a point $x_{0} \in X$ if there exists a continuous linear map $f^{\prime}\left(x_{0}\right): X \rightarrow Y$ so that

$$
\left\|f(x)-f\left(x_{0}\right)-f^{\prime}\left(x_{0}\right)\left(x-x_{0}\right)\right\|_{Y}=o\left(\left\|x-x_{0}\right\|_{X}\right)
$$

as $x \rightarrow x_{0}$ where $f^{\prime}\left(x_{0}\right) \in \mathcal{L}(X, Y)$ is called the Frechet derivative of $f$ at $x_{0}$. $f$ is said twice differentiable at $x_{0}$ if $f$ is differentiable at each point in 
a neighborhood of $x_{0}$ and $x \rightarrow f^{\prime}(x) \in \mathcal{L}(X, Y)$ is differentiable at $x_{0}$; and so on.

A map $f: U \subset X \rightarrow Y, U$ open, is analytic in $U$ if $f$ is infinitely often differentiable at each point of $U$ and if, for each $x \in U$, there exists $\delta=\delta(x)>0$ so that whenever $\|h\|_{X} \leq \delta$,

$$
f(x+h)=\sum_{k=0}^{\infty} \frac{1}{k !} f^{(k)}(x)\left[h^{k}\right]
$$

the series converging in $Y$-norm uniformly in $\|h\|_{X} \leq \delta$.

Consider the IVP for the generalized KdV equation

$$
\left\{\begin{array}{l}
\partial_{t} u+\partial_{x}(a(u))+\partial_{x}^{3} u=0, \quad x, t \in R \\
u(x, 0)=\psi(x)
\end{array}\right.
$$

where $a \in C^{\infty}(R ; R)$ with $a(0)=a^{\prime}(0)=0$.

According to Theorem 3.1, for any $\phi \in H^{s}(R), s>3 / 4$, there is a $T>0$ and a neighborhood $U$ of $\phi$ in $H^{s}(R)$ such that (4.1) defines a nonlinear map $K$ from $U$ to $X_{l, r}^{T, s}$

$$
u:=K(\psi)
$$

for any $\psi \in U$ where $u$ is the solution of (3.1). We shall show in this section that $K$ is infinitely many times Frechet differentiable from $U$ to $X_{l, r}^{T, s}$.

If we suppose that the map $K$ is $n$ times Frechet differentiable, then its $n$-th order derivative $K^{(n)}(\psi)$ at $\psi \in U$ is a symmetric $n$-linear map from $H^{s}(R)$ to $X_{l, r}^{T, s}$ and for any $h_{1}, \ldots, h_{n} \in H^{s}(R)$,

$$
K^{(n)}(\phi)\left[h_{1}, \ldots, h_{n}\right]=\left\{\frac{\partial^{n}}{\partial \xi_{1} \ldots \partial \xi_{n}} K\left(\psi+\sum_{k=1}^{n} \xi_{k} h_{k}\right)\right\}_{0, \ldots, 0} .
$$

As for the homogeneous polynomial $K^{(n)}(\psi)\left[h^{n}\right]$ of degree $n$ induced by $K^{(n)}(\psi)$, it is given by

$$
K^{(n)}(\psi)\left[h^{n}\right]=\left\{\frac{d^{n}}{d \xi^{n}} K(\psi+\xi h)\right\}_{\xi=0}
$$


for any $h \in H^{s}(R)$.

Denote by

$$
w_{[1, \ldots, n]}^{(n)}=K^{(n)}(\psi)\left[h_{1}, \ldots, h_{n}\right] \quad \text { and } y_{n}=K^{(n)}(\psi)\left[h^{n}\right] .
$$

Then direct computation show that $w_{[1, \ldots, n]}^{(n)}$ solves

$$
\left\{\begin{array}{l}
\partial_{t} w_{[1]}^{(1)}+\partial_{x}\left(a^{\prime}(u) w_{[1]}^{(1)}\right)+\partial_{x}^{3} w_{[1]}^{(1)}=0 \\
w_{[1]}^{(1)}(x, 0)=h_{1}
\end{array}\right.
$$

for $n=1$ and

$$
\left\{\begin{array}{l}
\partial_{t} w_{[1, \ldots, n]}^{(n)}+\partial_{x}\left(a^{\prime}(u) w_{[1, \ldots, n]}^{(n)}\right)+\partial_{x}^{3} w_{[1, \ldots, n]}^{(n)}=-\partial_{x}\left(H_{n}\right) \\
w_{[1, \ldots, n]}^{(n)}(x, 0)=0
\end{array}\right.
$$

for $n \geq 2$ with $u=K(\psi)$ and

$$
H_{n}=\sum_{j=2}^{n} \frac{a^{(j)}}{j !} \sum_{k_{1}+\ldots+k_{j}=n} \sum_{\mho} w_{\left[i_{1}^{1}, \ldots, i_{k_{1}}^{1}\right]}^{\left(k_{1}\right)} w_{\left[i_{1}^{2}, \ldots, i_{k_{2}}^{2}\right]}^{\left(k_{2}\right)} \ldots w_{\left[i_{1}^{j}, \ldots, i_{k_{j}}^{j}\right]}^{\left(k_{j}\right)}
$$

where $\sum_{\mho}$ is the summation over all $\left(i_{1}^{1}, \ldots, i_{k_{1}}^{1}, \ldots i_{1}^{j}, \ldots, i_{k_{j}}^{j}\right)$ satisfying

$$
1 \leq i_{1}^{m}<i_{2}^{m}<\ldots<i_{k_{m}}^{m} \leq n
$$

for $m=1,2, \ldots, j$ and

$$
\bigcup_{m=1}^{j} \bigcup_{l=1}^{k_{m}}\left\{i_{l}^{m}\right\}=\{1,2, \ldots, n\}
$$

As for $y_{n}$, it solves

$$
\left\{\begin{array}{l}
\partial_{t} y_{1}+\partial_{x}\left(a^{\prime}(u) y_{1}\right)+\partial_{x}^{3} y_{1}=0 \\
y_{1}(x, 0)=h
\end{array}\right.
$$

for $n=1$ and

$$
\left\{\begin{array}{l}
\partial_{t} y_{n}+\partial_{x}\left(a^{\prime}(u) y_{n}\right)+\partial_{x}^{3} y_{n}=-\partial_{x}\left(M_{n}\right) \\
y_{n}(x, 0)=0
\end{array}\right.
$$


for $n \geq 2$ where

$$
M_{n}=\sum_{j=2}^{n} \frac{a^{(j)}(u)}{j !} \sum_{k_{1}+\ldots+k_{j}=n} \frac{n !}{k_{1} ! \ldots k_{j} !} y_{k_{1}} . . y_{k_{j}} .
$$

On the other hand, according to Theorem 2.1, given $u \in X_{0,0}^{T, s}$ with $s>3 / 4,(4.2)$ defines a linear $\operatorname{map} \mathcal{K}^{(1)}(u)$ from $H^{s}(R)$ to $X_{l, r}^{T, s}$ with $(l, r) \in$ $\left[0, s-\frac{3}{4}\right] \times\left[0, s-\frac{3}{4}\right)$,

$$
\mathcal{K}^{(1)}(u)\left[h_{k}\right]=w_{[k]}^{(1)}
$$

where $w_{[k]}^{(1)}$ is the solution of (4.2) with the initial value $h_{k} \in H^{s}(R), k=$ $1,2 \ldots$ Inductively, (4.2)-(4.3) defines an n-linear map $\mathcal{K}^{(n)}(u)$ from the n-fold space $\left(H^{s}(R)\right)^{n}$ to $X_{l, r}^{T, s}$ for any $n \geq 2$.

Proposition 4.1 Let $s>3 / 4,(l, r) \in\left[0, s-\frac{3}{4}\right] \times\left[0, s-\frac{3}{4}\right), T>0$ and $u \in X_{0,0}^{T, s}$ be given. Then for any $h_{1}, \ldots, h_{n} \in H^{s}(R)$, (4.2)-(4.3) has a unique solution $w_{[1, . ., n]}^{(n)}$, which defines an $n$-linear map $\mathcal{K}^{(n)}(u)$ from the $n$ fold space $\left(H^{s}(R)\right)^{n}$ to $X_{l, r}^{T, s}$. Moreover,

$$
\left\|\mathcal{K}^{(n)}(u)\left[h_{1}, \ldots, h_{n}\right]\right\|_{X_{l, r}^{T, s}} \leq c\left(n,\|u\|_{X_{0,0}^{T, s}}\right) \prod_{k=1}^{n}\left\|h_{k}\right\|_{s}
$$

for any $n \geq 1$ and $h_{1}, \ldots, h_{n} \in H^{s}(R)$ where $c(n,$.$) is a continuous monotone$ increasing function from $R^{+}$to $R^{+}$with $c(n, 0)=0$.

Proof: It is easy to see that (4.7) is true with $n=1$ by applying (2.19) to (4.2). Similarly, for $n=2$, applying (2.19) to (4.3) yields

$$
\left\|w_{[1,2]}^{(2)}\right\|_{X_{l, r}^{T, s}} \leq \beta\left(\|u\|_{X_{0,0}^{T, s}}\right) \int_{-T}^{T}\left\|\partial_{x} H_{2}\right\|_{s} d \tau
$$

Then it follows from Lemma 2.8 that

$$
\begin{aligned}
\int_{-T}^{T}\left\|\partial_{x} H_{2}\right\|_{s} d t & =\int_{-T}^{T}\left\|\partial_{x}\left(\frac{a^{(2)}(u)}{2} w_{[1]}^{(1)} w_{[2]}^{(1)}\right)\right\|_{s} d t \\
& \leq c^{2} \beta_{2}\left(\|u\|_{X_{0,0}^{T, s}}\right)\left\|w_{[1]}^{(1)}\right\|\left\|_{X_{0,0}^{T, s}}\right\| w_{[2]}^{(1)} \|_{X_{0,0}^{T, s}} \\
& \leq c^{2} \beta_{2}\left(\|u\|_{X_{0,0}^{T, s}}\right) c^{2}\left(1,\|u\|_{X_{0,0}^{T, s}}\right)\left\|h_{1}\right\|_{s}\left\|h_{2}\right\|_{s}
\end{aligned}
$$


Thus,

$$
\begin{aligned}
\left\|w_{[1,2]}^{(2)}\right\|_{X_{l, r}^{T, s}} & \leq c^{2} \beta\left(\|u\|_{X_{0,0}^{T, s}}\right) \beta_{2}\left(\|u\|_{X_{0,0}^{T, s}}\right) c^{2}\left(1,\|u\|_{X_{0,0}^{T, s}}\right)\left\|h_{1}\right\|_{s}\left\|h_{2}\right\|_{s} \\
& :=c\left(2,\|u\|_{X_{0,0}^{T, s}}\right)\left\|h_{1}\right\|\left\|_{s}\right\| h_{2} \|_{X_{0,0}^{T, s}}
\end{aligned}
$$

Assume that (4.7) is true for $1 \leq k \leq N-1$. Applying (2.19) to (4.3) with $n=N$ yields

$$
\begin{aligned}
& \left\|w_{[1, \ldots, N]}^{(N)}\right\|_{X_{l, r}^{T, s}} \leq \beta\left(\|u\|_{X_{0,0}^{T, s}}\right) \int_{-T}^{T}\left\|\partial_{x} H_{N}\right\|_{s} d t \\
& \leq \beta\left(\|u\|_{\left.X_{0,0}^{T, s}\right)} \sum_{j=2}^{N} \frac{c^{j}}{j !} \beta_{j}\left(\|u\|_{X_{0,0}^{T, s}}\right) \sum_{k_{1}+\ldots+k_{j}=N} \prod_{l=1}^{j}\left\|w_{\left[i_{1}^{l}, \ldots, i_{k_{l}}^{l}\right]}^{\left(k_{l}\right)}\right\|_{X_{0,0}^{T, s}}\right. \\
& \leq \beta\left(\| u \| _ { X _ { 0 , 0 } ^ { T , s } ) } \sum _ { j = 2 } ^ { N } \frac { c ^ { j } } { j ! } \beta _ { j } \left(\| u \| _ { X _ { 0 , 0 } ^ { T , s } ) } \sum _ { k _ { 1 } + \ldots + k _ { j } = N } \prod _ { l = 1 } ^ { j } c \left(k_{l},\|u\|_{\left.X_{0,0}^{T, s}\right)}\left\|h_{\left[i_{1}^{l}, \ldots, i_{k_{l}}^{l}\right]}\right\|_{s}\right.\right.\right. \\
& :=c\left(N,\|u\|_{X_{0,0}^{T, s}}\right) \prod_{k=1}^{N}\left\|h_{k}\right\|_{s} .
\end{aligned}
$$

The proof is completed by induction.

Corollary 4.1 Let $s>3 / 4,(l, r) \in\left[0, s-\frac{3}{4}\right] \times\left[0, s-\frac{3}{4}\right), T>0$ and $u \in X_{0,0}^{T, s}$ be given. Then (4.5)-(4.6) defines a homogeneous polynomial $\mathcal{K}^{(n)}(u)\left[h^{n}\right]$ of degree $n$ from $H^{s}(R)$ to $X_{l, r}^{T, s}$ and

$$
\left\|\mathcal{K}^{(n)}(u)\left[h^{n}\right]\right\|_{X_{l, r}^{T, s}} \leq c\left(n,\|u\|_{X_{0,0}^{T, s}}\right)\|h\|_{s}^{n}
$$

for any $h \in H^{s}(R)$.

Now we may define formally the n-th Taylor polynomial $P_{n}(h)$ of the map $K$ at some $\phi \in H^{s}(R)$ as

$$
\begin{aligned}
P_{n}(h) & =K(\phi)+\sum_{k=1}^{n} \frac{1}{k !} \mathcal{K}^{(k)}(u)\left[h^{k}\right] \\
& :=u+\sum_{k=1}^{n} \frac{1}{k !} y_{k}
\end{aligned}
$$

where $u=K(\phi)$. 
Proposition 4.2 Let $z_{n}$ denote the $n$-th Taylor remainder of $K$ at $\phi \in$ $H^{s}(R)$, i.e.

$$
z_{n}=K\left((\phi+h)-P_{n}(h)\right.
$$

Then it solves

$$
\left\{\begin{array}{l}
\partial_{t} z_{0}+\partial_{x}\left(F_{1}(u, v) z_{0}\right)+\partial_{x}^{3} z_{0}=0 \\
z_{0}(x, 0)=h(x)
\end{array}\right.
$$

for $n=0$ and

$$
\left\{\begin{array}{l}
\partial_{t} z_{n}+\partial_{x}\left(F_{1}(u, v) z_{n}\right)+\partial_{x}^{3} z_{n}=-\partial_{x}\left(D_{n}\right) \\
z_{n}(x, 0)=0
\end{array}\right.
$$

for $n \geq 1$ where

$$
u=K(\phi), \quad v=K(\phi+h)
$$

and

$$
D_{n}=\sum_{m=2}^{n+1} F_{m}(u, v) \sum_{k=0}^{n+1-m} z_{k} \sum_{k_{1}+\ldots+k_{m-1}=n-k} q_{k_{1}} \ldots q_{k_{m-1}}
$$

with $F_{m}(u . v)$ defined by (1.14) and

$$
q_{m}=\frac{y_{m}}{m !}
$$

for $m=1, \ldots, n+1$.

Proof: Direct computation shows easily that (4.9) and (4.10) with $n=1$ are true. Assume that (4.10) is true for $n=N$. Then, for $n=N+1$, by definition,

$$
z_{N+1}=z_{N}-\frac{1}{(N+1) !} y_{N+1}=z_{N}-q_{N+1}
$$

where $q_{N+1}$ solves

$$
\left\{\begin{array}{l}
\partial_{t} q_{N+1}+\partial_{x}\left(a^{\prime}(u) q_{N+1}\right)+\partial_{x}^{3} q_{N+1}=-\partial_{x}\left(E_{N+1}\right) \\
q_{N+1}=0
\end{array}\right.
$$

with 


$$
E_{N+1}=\sum_{m=2}^{N+1} \frac{a^{(m)}(u)}{m !} \sum_{k_{1}+\ldots+k_{m}=N+1} q_{k_{1}} \ldots q_{k_{m}} .
$$

Hence

$$
\partial_{t} z_{N+1}+\partial_{x}^{3} z_{N+1}=-\partial_{x}\left(F_{1}(u, v) z_{N}-a^{(1)}(u) q_{N+1}\right)-\partial_{x}\left(G_{N+1}\right)
$$

where

$$
\begin{aligned}
G_{N+1} & =\sum_{m=2}^{N+1} F_{M}(u, v) \sum_{k=0}^{N+1-m} z_{k} \sum_{k_{1}+\ldots+k_{M_{1}}=N-k} q_{k_{1}} \ldots q_{k_{m-1}}- \\
& -\sum_{m=1}^{N+1} \frac{a^{(m)}(u)}{m !} \sum_{k_{1}+\ldots+k_{m}=N+1} q_{k_{1}} \ldots q_{k_{m}} .
\end{aligned}
$$

Note that

$$
\begin{gathered}
F_{1}(u, v) z_{N}-a^{\prime}(u) q_{N+1}=F_{1}(u, v)\left(z_{N}-q_{N+1}\right)+\left(F_{1}(u, v)-a^{\prime}(u)\right) q_{N+1} \\
=F_{1}(u, v) z_{N+1}+F_{2}(u, v) z_{0} q_{N+1}, \\
\sum_{m=2}^{N+1} F_{m}(u, v) \sum_{k=0}^{N+1-m} z_{k} \sum_{k_{1}+\ldots+k_{m-1}=N-k} q_{k_{1}} \ldots q_{k_{m-1}} \\
=\sum_{m=2}^{N+1} F_{m}(u, v) \sum_{k=0}^{N+1-m}\left(z_{k}-q_{k+1}\right) * \sum_{k_{1}+\ldots+k_{m-1}=N_{k}} q_{k_{1}} \ldots q_{k_{m-1}}+ \\
+\sum_{m=2}^{N+1} F_{m}(u, v) \sum_{k=0}^{N+1-m} q_{k+1} \sum_{k_{1}+\ldots+k_{m-1}=N-k} \ldots q_{k_{m-1}} \\
=\sum_{m=2}^{N+1} F_{m}(u, v) \sum_{k=1}^{N+2-m} z_{k} q_{k_{1}+\ldots+k_{m-1}=N+1-k} q_{k_{m-1}}+ \\
+\sum_{m=2}^{N+1} F_{m}(u, v) \sum_{k_{1}+\ldots+k_{m}=N+1} q_{k_{1}} \ldots q_{k_{m}}
\end{gathered}
$$

and

$$
\sum_{m=2}^{N+1} F_{m}(u, v) \sum_{k_{1}+\ldots+k_{m}=N+1} q_{k_{1}} \ldots q_{k_{m}}-\sum_{m=2}^{N+1} \frac{a^{(m)}(u)}{m !} \sum_{k_{1}+\ldots+k_{m}=N+1} q_{k_{1}} \ldots q_{k_{m}}
$$




$$
\begin{aligned}
& =\sum_{m=2}^{N+1}\left(F_{m}(u, v)-\frac{a^{(m)}(u)}{m !}\right)_{k_{1}+\ldots+k_{m}=N+1} q_{k_{1}} \ldots q_{k_{m}} \\
& =\sum_{m=2}^{N+1} F_{m+1}(u, v) z_{0} \sum_{k_{1}+k_{2}+\ldots+k_{m}=N+1} q_{k_{1}} \ldots q_{k_{m}} \\
& =\sum_{m=3}^{N+2} F_{m}(u, v) z_{0} \sum_{k_{1}+\ldots+k_{m-1}=N+1} q_{k_{1}} \ldots q_{k_{m-1}} .
\end{aligned}
$$

Thus

$$
\begin{gathered}
F_{1}(u, v) z_{n}-a^{\prime}(u) q_{N+1}-G_{N+1}=F_{1}(u, v) z_{N+1}+F_{2}(u, v) z_{0} q_{N+1}+ \\
+\sum_{m=2}^{N+1} F_{m}(u, v) \sum_{k=1}^{N+2-m} z_{k} \sum_{k_{1}+. .+k_{m-1}=N+1-k} q_{k_{1}} \ldots q_{k_{m-1}}+ \\
\quad+\sum_{m=3}^{N+2} F_{m}(u, v) z_{0} \sum_{k_{1}+\ldots+k_{m-1}=N+1} q_{k_{1}} \ldots q_{k_{m-1}} \\
=F_{1}(u, v) z_{N+1}+\sum_{m=2}^{N+2} F_{m}(u, v) \sum_{k=0}^{N+2-m} z_{k} \sum_{k_{1}+\ldots+k_{m-1}=N+1-k} q_{k_{1}} \ldots q_{k_{m-1}}
\end{gathered}
$$

and we conclude that

$$
\left\{\begin{array}{l}
\partial_{t} z_{N+1}+\partial_{x}\left(F_{1}(u, v) z_{N+1}\right)+\partial_{x}^{3} z_{N+1}=-\partial_{x}\left(D_{N+1}\right) \\
z_{N+1}(x, 0)=0
\end{array}\right.
$$

which is (4.10) with $n=N+1$. The proof is completed by induction.

Theorem 4.1 Let $s>\frac{3}{4}$ and $(l, r) \in\left[0, s-\frac{3}{4}\right] \times\left[0, s-\frac{3}{4}\right)$ be given. Then, for any $\phi^{*} \in H^{s}(R)$, there exist a $T>0$ and a neighborhood $U$ of $\phi^{*}$ in $H^{s}(R)$ such that the nonlinear map $K$ defined by the IVP (3.1) is infinitely many time Frechet differentiable in $U$ from $H^{s}(R)$ to $X_{l, r}^{T, s}$. Its $n$-th derivative $K^{(n)}$ at $\psi \in U$ is given by

$$
K^{(n)}(\psi)\left[h_{1}, \ldots, h_{n}\right]=\mathcal{K}^{(n)}(u)\left[h_{1}, \ldots, h_{n}\right]
$$

for any $h_{1}, \ldots, h_{n} \in H^{s}(R)$ where $\mathcal{K}^{(n)}(u)$ is defined by (4.2) and (4.3) and $u=K(\psi)$. 
Proof: We only need to prove that for any $\psi \in U$,

$$
K(\phi+h)=\sum_{k=0}^{n} \frac{1}{k !} \mathcal{K}^{(k)}(\phi)\left[h^{k}\right]+o\left(\|h\|_{s}^{n}\right)
$$

as $h \rightarrow 0$ in $H^{s}(R)$ uniformly for $\|\phi-\psi\|_{s} \leq\|h\|_{s}$ by the Converse Taylor Theorem (see [8]).

Let

$$
v=K(\phi+h), \quad u=K(\phi), \quad y^{(k)}=\mathcal{K}^{(k)}(u)\left[h^{k}\right]
$$

for $1 \leq k \leq n$ and

$$
z_{0}=v-u, \quad z_{1}=z_{0}-y_{0}, \quad z_{n}=z_{n-1}-\frac{1}{n !} y_{n}
$$

Then, by Proposition 4.2,

$$
\left\{\begin{array}{l}
\partial_{t} z_{0}+\partial_{x}\left(F_{1}(u, v) z_{0}\right)+\partial_{x}^{3} z_{0}=0 \\
z_{0}(x, 0)=h
\end{array}\right.
$$

and for $n \geq 1$

$$
\left\{\begin{array}{l}
\partial_{t} z_{n}+\partial_{x}\left(F_{1}(u, v) z_{n}\right)+\partial_{x}^{3} z_{n} F_{1}(u, v)=-\partial_{x} D_{n} \\
z_{n}(x, 0)=0
\end{array}\right.
$$

Choose $\delta_{1}>0$ such that

$$
S_{\delta_{1}}(\psi)=\left\{\phi \in H^{s}(R),\|\phi-\psi\|_{s} \leq \delta_{1}\right\} \subset U \text {. }
$$

Obviously, $K(\phi)$ is bounded on $S_{\delta_{1}}(\psi)$ since $K$ is a continuous map.

By Corollary 4.1,

$$
\left\|q_{k}\right\|_{X_{l, r}^{T, s}} \leq c\left(k,\|u\|_{X_{0,0}^{T, s}}\right)\|h\|_{s}^{k}, \quad k=1,2, \ldots, n
$$

where $u=K(\phi)$ and $c\left(k,\|u\|_{X_{0,0}^{T, s}}\right)$ is uniformly bounded on $S_{\delta_{1}}(\psi)$. It suffices to prove that

$$
\left\|z_{n}\right\|_{X_{l, r}^{T, s}} \leq \gamma_{n}\|h\|_{s}^{n+1}
$$


for $n \geq 0$ where $\gamma_{n}$ is uniformly bounded for $\phi \in S_{\delta_{1}}(\psi)$. Indeed, it is easy to obtain (4.15) for $n=0$ by using Theorem 2.1 to (4.13) and if we suppose (4.15) is true for $n \leq N$, then applying Theorem 2.1 and Lemma 2.8 to (4.14) with $n=N+1$, we have that

$$
\begin{aligned}
& \left\|z_{N+1}\right\|_{X_{l, s}^{T, s}} \\
& \leq c \sum_{m=2}^{N+2} \sum_{k=0}^{N+2-m} \sum_{k_{1}+\ldots+k_{m-1}=N+1-k} \int_{-T}^{T}\left\|\partial_{x}\left(F_{m}(u, v) z_{k} q_{k_{1}} \ldots q_{k_{m-1}}\right)\right\|_{s} d t \\
& \leq c \sum_{m=2}^{N+2} \sum_{k=0}^{N+2-m} \sum_{k_{1}+\ldots+k_{m-1}=N+1-k} c^{m} \beta_{m} \Lambda_{0,0}^{s}\left(T ; z_{k}\right) \prod_{j=1}^{m-1} \Lambda_{0,0}^{s}\left(T ; q_{k_{j}}\right) \\
& \leq c \sum_{m=2}^{N+1} \sum_{k=0}^{N+2-m} \sum_{k_{1}+\ldots+k_{m-1}=N+1-k} c^{m} \gamma_{m} \beta_{m} \prod_{j=1}^{m-1} c\left(k_{j},\|u\|_{X_{0,0}^{T, s}}\right)\|h\|_{s}^{N+2} \\
& :=\gamma_{N+1}\|h\|_{s}^{N+2}
\end{aligned}
$$

The proof is completed by induction.

Corollary 4.2 (Taylor's Formula) For any $\phi \in U$ and $h \in H^{s}(R)$ satisfying

$$
\begin{gathered}
\phi+\xi h \in U, \quad \text { for any } \xi \in(0,1), \\
K(\phi+h)=\sum_{j=0}^{n-1} \frac{1}{j !} K^{(j)}(\phi)\left[h^{j}\right]+\int_{0}^{1} \frac{(1-\xi)^{n-1}}{n !} K^{(n)}(\phi+\xi h)\left[h^{n}\right] d \xi
\end{gathered}
$$

with any $n \geq 1$.

Proof: See [7, Theorem 8.14.3].

\section{Analyticity}

In the previous section we proved the map $K$ is infinitely many times Frechet differentiable from $H^{s}(R)$ to $X_{l, r}^{T, s}$. Naturally the further question is whether the map $K$ is an analytic map, i.e., whether it has Taylor series expansion at any $\phi \in H^{s}(R)$ :

$$
K(\phi+h)=\sum_{n=0}^{\infty} \frac{K^{(n)}(\phi)}{n !}\left[h^{n}\right]
$$


where the series coverges in $X_{l, r}^{T, s}$ uniformly for $\|h\|_{s} \leq \delta$ with some $\delta>0$ only depending on $\phi$. For that purpose we need a better estimate of the n-th derivative $y_{n}=K^{(n)}(\phi)\left[h^{n}\right]$ of the map $K$ at $\phi$ and the n-th Taylor remainder $z_{n}$.

Proposition 5.1 Let $u \in X_{0,0}^{T, s}$ be given and $y_{n}$ is the solution of (4.5)(4.6). Then there exists a sequence $\alpha(n)$ given by

$$
\alpha(1)=1
$$

and

$$
\alpha(n)=\sum_{j=2}^{n} \frac{\beta_{j}}{j !} \sum_{k_{1}+\ldots+k_{j}=n} \alpha\left(k_{1}\right) \alpha\left(k_{2}\right) \ldots \alpha\left(k_{j}\right)
$$

for $n \geq 2$ such that

$$
\left\|y_{n}\right\|_{X_{l, r}^{T, s}} \leq c^{n} n ! \alpha(n)\|h\|_{s}^{n}
$$

for any $n \geq 1$ where $c>0$ is a constant independt of $n$ and $h$,

$$
\beta_{j}=\beta_{j}\left(\|u\|_{X_{0,0}^{T, s}}\right.
$$

is a continuous monotone function depending only on $a^{(j)}$, and $\beta_{j} \equiv 0$ if $\alpha^{(j)} \equiv 0$.

Proof: Denote by $q_{n}=y_{n} / n$ !. It solves

$$
\left\{\begin{array}{l}
\partial_{t} q_{1}+\partial_{x}\left(a^{\prime}(u) q_{1}\right)+\partial_{x}^{3} q_{1}=0 \\
q_{1}(x, 0)=h(x)
\end{array}\right.
$$

for $n=1$ and

$$
\left\{\begin{array}{l}
\partial_{t} q_{n}+\partial_{x}\left(a^{\prime}(u) q_{n}\right)+\partial_{x}^{3} q_{n}=-\partial_{x}\left(\sum_{j=2}^{n} \frac{a^{(j)}(u)}{j !} \sum_{k_{1}+\ldots+k_{j}=n} q_{k_{1}} \ldots q_{k_{j}}\right) \\
q_{n}(x, 0)=0
\end{array}\right.
$$

for $n \geq 2$.

Applying (2.19) to (5.5) yields

$$
\left\|q_{1}\right\|_{X_{l, r}^{T, s}} \leq c\|h\|_{s}
$$


Assume that

$$
\left\|q_{m}\right\|_{X_{l, r}^{T, s}} \leq c^{2 m-1} \alpha(m)\|h\|_{s}^{m}, \quad \text { for } 1 \leq m \leq N
$$

Then applying (2.19) to (5.6) with $n=N+1$, we obtain

$$
\begin{aligned}
\left\|q_{N+1}\right\|_{X_{l, s}^{T, s}} & \leq c \sum_{j=2}^{N+1} \frac{1}{j !} \sum_{k_{1}+\ldots+k_{j}=N+1} \int_{-T}^{T}\left\|\partial_{x}\left(a^{(j)}(u) q_{k_{1}} \ldots q_{k_{j}}\right)\right\|_{s} d t \\
& \leq c \sum_{j=2}^{N+1} \frac{\beta_{j}}{j !} \sum_{k_{1}+\ldots+k_{j}=N+1} \prod_{l=1}^{j}\left\|q_{k_{l}}\right\|_{X_{0,0}^{T, s}} \\
& \leq c \sum_{j=2}^{N+1} \frac{\beta_{j}}{j !} \sum_{k_{1}+\ldots+k_{j}=N+1} \prod_{l=1}^{j} c^{2 k_{l}-1}\|h\|_{s}^{k_{l}} \alpha\left(k_{l}\right) \\
& \leq c^{2(N+1)-1} \alpha(N+1)\|h\|_{s}^{N+1} .
\end{aligned}
$$

Thus we have proved by induction that

$$
\left\|y_{n}\right\|_{X_{l, r}^{T, s}} \leq c^{2 n-1} n ! \alpha(n)\|h\|_{s}^{n}
$$

which is (5.4) with a different $c>0$. The proof is completed by induction.

Proposition 5.2 Let $s>3 / 4, T>0,(l, r) \in\left[0, s-\frac{3}{4}\right] \times\left[0, s-\frac{3}{4}\right)$ and $u, v \in X_{0,0}^{T, s}$ be given. If $z_{n}$ is the solution of (4.9)-(4.10) with $y_{n}$ being the solution of (4.5)-(4.6), then there exists a sequence $\gamma(n)$ given by

$$
\begin{gathered}
\gamma(0)=\eta_{1} \\
\gamma(n)=\eta_{1} \sum_{m=2}^{n+1} \eta_{m} \sum_{k=0}^{n+1-m} \gamma(k) \sum_{k_{1}+\ldots+k_{m-1}=n-k} \prod_{i=1}^{m-1} c^{i} \alpha(i)
\end{gathered}
$$

for $n \geq 1$ with $\alpha(n)$ given by (5.2)-(5.3) such that

$$
\left\|z_{n}\right\|_{X_{l, r}^{T, s}} \leq \gamma(n)\|h\|_{s}^{n+1}, \quad n \geq 0
$$

where

$$
\eta_{j}=\eta_{j}\left(\|u\|_{X_{0,0}^{T, s}},\|v\|_{X_{0,0}^{T, s}}\right),
$$

depending only on $a^{(j)}$, is a continuous function of $\|u\|_{X_{0,0}^{T, s}}$ and $\|v\|_{X_{0,0}^{T, s}}$ with $\eta_{j} \equiv 0$ if $\alpha^{(j)} \equiv 0$, and $c>0$ is a constant independent of $n$ and $h$. 
Proof: It follows from (4.9) by applying (2.19) that

$$
\left\|z_{0}\right\|_{X_{l, r}^{T, s}} \leq \eta_{1}\|h\|_{s}
$$

Assume that (5.9) is true for all $0 \leq n \leq N-1$. Then applying (2.19) and (2.17) to (4.10) with $n=N$, we obtain that

$$
\begin{aligned}
\left\|z_{N}\right\|_{X_{l, r}^{T, s}} & \leq \eta_{1} \sum_{m=2}^{N+1} \sum_{k=0}^{N+1-m} \sum_{k_{1}+\ldots+k_{m_{1}}=N-k} \int_{-T}^{T}\left\|\partial_{x}\left(F_{m} z_{k} \prod_{i=1}^{m-1} q_{k_{i}}\right)\right\|_{s} d t \\
& \leq \eta_{1} \sum_{m=2}^{N+1} \sum_{k=0}^{N+1-m} \sum_{k_{1}+\ldots+k_{m-1}=N-k} \eta_{m}\left\|z_{k}\right\|_{X_{0,0}^{T, s}} \prod_{i=1}^{m-1}\left\|q_{k_{i}}\right\|_{s} \\
& \leq \eta_{1} \sum_{m=2}^{N+1} \sum_{k=0}^{N+1-m} \sum_{k_{1}+\ldots+k_{m-1}=N-k} \eta_{m} \gamma(k)\|h\|_{s}^{N+1} \prod_{i=1}^{m-1} c^{i} \alpha(i) \\
& \leq \gamma(N)\|h\|_{s}^{N+1} .
\end{aligned}
$$

The proof is completed by induction.

Now we consider the map $K$ defined by (3.1) from an open set $U$ in $H^{s}(R)$ to $X_{l, r}^{T, s}$ where $s>3 / 4$ and $(l, r) \in\left[0, s-\frac{3}{4}\right] \times\left[0, s-\frac{3}{4}\right)$.

Proposition 5.3 Let $\phi \in U$. If there is a $\delta_{1}>0$ and $c>0$ such that

$$
S_{\delta_{1}}(\phi)=\left\{\psi \in H^{s}(R) \mid \quad\|\phi-\psi\|_{s}<\delta_{1}\right\} \subset U
$$

and

$$
\gamma(n) \leq c^{n}, \quad n \geq 0
$$

uniformly for $\psi \in S_{\delta_{1}}(\phi)$ where $\gamma(n)$ is defined by (5.7)-(5.8) with $u=K(\phi)$ and $v=K(\psi)$, then there exists a $\delta>0$ such that the series (5.1) coverges in $X_{l, r}^{T, s}$ uniformly for $\|h\|_{s} \leq \delta$.

Proof: Consider the n-th Taylor remainder

$$
z_{n}=K(\phi+h)-\sum_{j=0}^{n} \frac{1}{j !} K^{(j)}(\phi)\left[h^{j}\right] .
$$

According to Proposition 5.2 and hypothesis (5.10),

$$
\left\|z_{n}\right\|_{X_{l, r}^{T, s}} \leq \gamma(n)\|h\|_{s}^{n+1} \leq c^{n}\|h\|_{s}^{n+1}
$$


where $c>0$ is independent of $n$ and $h \in H^{s}(R)$ with $\|h\|_{s}<\delta_{1}$. Thus if we choose $\delta>0$ such that

$$
\delta<\frac{1}{2 c_{1}}
$$

then

$$
\left\|z_{n}\right\|_{X_{l, r}^{T, s}} \leq\left(\frac{1}{2}\right)^{n}, \quad n \geq 1
$$

for any $h \in H^{s}(R)$ with $\|h\|_{s} \leq \delta$. The proof is completed.

In the following we shall show that if $a(u)$ in $(3.1)$ is a polynomial of degree $N$, i.e.

$$
a(u)=\sum_{j=2}^{N} b_{j} u^{j}
$$

with $b_{j}, j=1,2 \ldots, N$ being real constants, then hypothesis (5.10) is satisfied for any $\phi \in U$ and therefore the map $K$ is an analytic map from $U \subset H^{s}(R)$ to $X_{l, r}^{T, s}$.

First we prove a technical lemma.

Lemma 5.1 Let $N \geq 1$ be a given integer and $\alpha_{n}$ be a sequence given by

$$
\begin{gathered}
\alpha_{N}(1)=1, \\
\alpha_{N}(n)=\sum_{j=2}^{n} \frac{b_{j}}{j !} \sum_{k_{1}+\ldots+k_{j}=n} \alpha_{N}\left(k_{1}\right) \ldots \alpha_{N}\left(k_{j}\right), \quad \text { for } 2 \leq n \leq N-1,
\end{gathered}
$$

and

$$
\alpha_{N}(n)=\sum_{j=2}^{N} \frac{b_{j}}{j !} \sum_{k_{1}+\ldots+k_{j}=n} \alpha_{N}\left(k_{1}\right) \ldots \alpha_{N}\left(k_{j}\right), \quad \text { for } n \geq N .
$$

where $b_{j}, 1 \leq j \leq N$ are given constants. Then there exists a constant $c>0$ such that

$$
\alpha_{N}(n) \leq c^{n}
$$

for any $n \geq 1$.

Remark 5.1 In the case $N=2$ and $b_{2}=1$, the lemma is Proposition 3.4 in [47] where it is shown that

$$
\alpha_{2}(n)=\frac{2^{n-1}(2 n-3) ! !}{n !} \quad \text { for any } n \geq 2 .
$$


Proof of Lemma 5.1: Note that that $\alpha_{N}(n)$, for any $n \geq 1$, is uniquelly determined by (5.12) and (5.13) inductively. In particular, we may obtain

$$
\alpha_{N}(1), \alpha_{N}(2), \ldots, \alpha_{N}(N-1)
$$

explicitly by computation.

Let $P_{N}(x)$ be a polynomial of degree $N$ as follows

$$
\begin{aligned}
P_{N}(x, y) & =y-\sum_{j=2}^{N} \frac{b_{j}}{j !}\left(\sum_{k=1}^{N-1} \alpha_{N}(k) x^{k}+y\right)^{j}- \\
& -\sum_{j=2}^{N} \frac{b_{j}}{j !} \sum_{k=j}^{N-1} x^{k} \sum_{k_{1}+\ldots+k_{j}=k} \alpha_{N}\left(k_{1}\right) \ldots \alpha_{N}\left(k_{j}\right) .
\end{aligned}
$$

It is easy to check that

$$
P_{N}(0,0)=0, \quad \frac{\partial}{\partial y} P_{N}(0,0)=1 .
$$

Thus, according to the implicit function theorem,

$$
P_{N}(x, y)=0
$$

has a unique solution

$$
y=f(x) \quad \text { with } f(0)=0
$$

in the neighborhood of $x=0$ such that

$$
P_{N}(x, f(x))=0, \quad \text { for any }|x| \leq \delta
$$

where $\delta>0$ is a constant. Besides, direct computation shows that $f^{(j)}(0)=$ 0 , for $j=1,2, \ldots, N-1$.

Moreover, $y=f(x)$ is a real analytic function in a neighborhood of $x=0$, i.e. $f(x)$ has a Taylor series expansion at $x=0$,

$$
y=f(x)=\sum_{j=N}^{\infty} d_{j} x^{j}
$$


which is uniformly convergent for $|x|<\delta$ with some $\delta>0$.

To see this, let

$$
g=\sum_{k=1}^{N-1} \alpha_{N}(k) x^{k}+y
$$

and

$$
z=\sum_{k=1}^{N-1} \alpha_{N}(k) x^{n}+\sum_{j=2}^{N} \frac{b_{j}}{j !} \sum_{k=j}^{N-1} x^{k} \sum_{k_{1}+\ldots+k_{j}=k} \alpha_{N}\left(k_{1}\right) \ldots \alpha_{N}\left(k_{j}\right):=h(x) .
$$

Then, equation (5.17) may be written as

$$
g-\sum_{j=2}^{N} \frac{b_{j}}{j !} g^{j}=z
$$

which obviously has an analytic solution $g(z)$ in a neiborhood of $z=0$ such that $g(0)=0$. Thus $y=f(x)=g(h(x))-\sum_{k=1}^{N-1} \alpha_{N}(k) x^{k}$ is an analytic function in a neighborhood of $x=0$ sicne $h(x)$ is a polynomial of $x$.

Plugging (5.18) into (5.17) and denoting by

$$
d_{j}=\alpha_{N}(j), \quad \text { for } j=1,2, \ldots, N-1,
$$

we have

$$
\begin{aligned}
y= & \sum_{j=N}^{\infty} b_{j} x^{j} \\
= & \sum_{j=2}^{N} \frac{b_{j}}{j !}\left(\sum_{k=1}^{N-1} \alpha_{N}(k) x^{k}+\sum_{k=N}^{\infty} d_{k} x^{k}\right)^{j}- \\
& \quad-\sum_{j=2}^{N} \frac{b_{j}}{j !} \sum_{k=j}^{N-1} x^{k} \sum_{k_{1}+\ldots+k_{j}=k} \alpha_{N}\left(k_{1}\right) \ldots \alpha_{N}\left(k_{j}\right) \\
= & \sum_{j=2}^{N} \frac{b_{j}}{j !}\left(\sum_{k=1}^{\infty} d_{k} x^{k}\right)^{j}-\sum_{k=2}^{N} \frac{b_{j}}{j !} \sum_{k=j}^{N-1} x^{k} \sum_{k_{1}+\ldots+k_{j}=k} d_{k_{1}} \ldots d_{k_{j}} \\
= & \sum_{j=2}^{N} \frac{b_{j}}{j !} \sum_{k=j}^{\infty} \sum_{k_{1}+\ldots+k_{j}=k} d_{k_{1}} x^{k_{1}} d_{k_{2}} x^{k_{2}} \ldots d_{k_{j}} x^{k_{j}}-
\end{aligned}
$$




$$
\begin{aligned}
& -\sum_{j=2}^{N} \frac{b_{j}}{j !} \sum_{k=j}^{N-1} \sum_{k_{1}+\ldots+k_{j}=k} d_{k_{1}} \ldots d_{k_{j}} x^{k} \\
= & \sum_{j=2}^{N} \frac{b_{j}}{j !}\left(\sum_{k=N}^{\infty} \sum_{k_{1}+\ldots+k_{j}=k} d_{k_{1}} \ldots d_{k_{j}}\right) x^{k} \\
= & \sum_{k=N}^{\infty}\left(\sum_{j=2}^{N} \frac{b_{j}}{j !} \sum_{k_{1}+\ldots+k_{j}=k} d_{K_{1}} \ldots d_{k_{j}}\right) x^{k}
\end{aligned}
$$

for any $|x|<\delta$. Thus we have

$$
d_{j}=\alpha_{N}(j), \quad j=1,2, \ldots, N-1
$$

and

$$
d_{k}=\sum_{j=2}^{N} \frac{b_{j}}{j !} \sum_{k_{1}+\ldots .+k_{j}=k} d_{k_{1}} \ldots d_{k_{j}}
$$

for any $k \geq N$. That is to say, $d_{j}$ for $j \geq 1$ also satisfy the induction relation (5.12)-(5.13). By uniqueness we have

$$
\alpha_{N}(k)=d_{k}, \quad \text { for any } k \geq 1 \text {. }
$$

On the other hand, $d_{k}, k \geq 1$ are coefficients of Taylor series (5.18) and therefore we must have

$$
d_{n} \leq c^{n}, \quad \text { for all } n \geq 1
$$

for some $c>0$ independent of $n$. The proof is completed.

Theorem 5.1 Let $s>3 / 4$ and $(l, r) \in\left[0, s-\frac{3}{4}\right] \times\left[0, s-\frac{3}{4}\right)$ be given and suppose that $a(u)$ is (3.1) is a polynomial of degree $N$. Then for any $\phi \in H^{s}(R)$, there is a $T>0$ and a neighborhood $U$ of $\phi$ in $H^{s}(R)$ such that the IVP (3.1) define an analytic map $K$ from $U$ to $X_{l, r}^{T, s}$, i.e., for any $\psi \in U$, there is a $\delta>0$ such that the Taylor series

$$
K(\psi+h)=K(\psi)+\sum_{n=1}^{\infty} \frac{1}{n !} K^{(n)}(\psi)\left[h^{n}\right]
$$

uniformly converges for $\|h\|_{s} \leq \delta$ in the space $X_{l, r}^{T, s}$. Moreover, if denote by

$$
u=K(\psi), \quad y_{n}=K^{(n)}(\psi)\left[h^{n}\right], \quad n \geq 1
$$


then

$$
\left\{\begin{array}{l}
\partial_{t} y_{1}+\partial_{x}\left(a^{\prime}(u) y_{1}\right)+\partial_{x}^{3} y_{1}=0 \\
y_{1}(x, 0)=h
\end{array}\right.
$$

for $n=1$ and

$$
\left\{\begin{array}{l}
\partial_{t} y_{n}+\partial_{x}\left(a^{\prime}(u) y_{n}\right)+\partial_{x}^{3} y_{n}=-\partial_{x} G_{N}(n) \\
y_{n}(x, 0)=0
\end{array}\right.
$$

for $n \geq 2$ where

$$
G_{N}(n)= \begin{cases}\sum_{j=2}^{n} \frac{a^{(j)}(u)}{j !} \sum_{k_{1}+\ldots+k_{j}=n} \frac{n !}{k_{1} ! \ldots k_{j} !} y_{k_{1}} \ldots y_{k_{j}} & \text { for } 2 \leq n \leq N-1 \\ \sum_{j=2}^{N} \frac{a^{(j)}(u)}{j !} \sum_{k_{1}+\ldots k_{j}=n} \frac{n !}{k_{1} ! \ldots k_{j} !} y_{k_{1}} \ldots y_{k_{j}} & \text { for } n \geq N .\end{cases}
$$

Proof: Since $U$ is an open subset in $H^{s}(R)$, there is a $\delta_{1}>0$ such that if $h \in H^{s}(R)$ with $\|h\|_{s} \leq \delta_{1}$, then

$$
\psi+h \in U \text {. }
$$

Denote by

$$
v=K(\psi+h), \quad u=K(\psi)
$$

and

$$
z_{0}=v-u, \quad z_{n}=z_{n-1}-\frac{1}{n !} y_{n}, \quad \text { for } n \geq 1 .
$$

Then, by proposition 5.1 and 5.2,

$$
\left\|y_{n}\right\|_{X_{l, r}^{T, s}} \leq n ! c^{n} \alpha(n)\|h\|_{s}^{n}
$$

and

$$
\left\|z_{n}\right\|_{X_{l, r}^{T, s}} \leq \gamma(n)\|h\|_{s}^{n+1} .
$$

Note that $a^{(j)}(u) \equiv 0$ for $j \geq N+1$ since $a(u)$ is a polynomial of degree $N$ by the assumption. Thus $\alpha(n)$ is given by

$$
\alpha(1)=1
$$




$$
\alpha(n)=\sum_{j=2}^{n} \frac{\beta_{j}}{j !} \sum_{k_{1}+\ldots+k_{j}=n} \alpha\left(k_{1}\right) \ldots \alpha\left(k_{j}\right)
$$

for $2 \leq n \leq N-1$ and

$$
\alpha(n)=\sum_{j-2}^{N} \frac{\beta_{j}}{j !} \sum_{k_{1}+\ldots+k_{j}=n} \alpha\left(k_{1}\right) \ldots \alpha\left(k_{j}\right)
$$

for $n \geq N$. Moreover,

$$
\begin{gathered}
\gamma(0)=\eta_{1} \\
\gamma(n)=\eta_{1} \sum_{j=2}^{n} \eta_{j} \sum_{k=0}^{n+1-j} \gamma(k) \sum_{k_{1}+\ldots k_{j-1}=n-k} \prod_{i=1}^{j-1} c^{k_{i}} \alpha\left(k_{i}\right)
\end{gathered}
$$

for $1 \leq n \leq N-1$ and

$$
\gamma(n)=\eta_{1} \sum_{j=2}^{N} \eta_{j} \sum_{k=0}^{n+1-j} \gamma(k) \sum_{k_{1}+\ldots k_{j-1}=n-k} \prod_{i=1}^{j-1} c^{k_{i}} \alpha\left(k_{i}\right)
$$

According to Proposition 5.3, we need to show that there is a $c_{*}>0$ such that

$$
\gamma(n) \leq c_{*}^{n}
$$

for any $n \geq N$ with $c_{*}$ independent of $n$ and $\|h\|_{s} \leq \delta_{1}$. To this end, we first see from Lemma 5.1 that

$$
\alpha(n) \leq c_{1}^{n}, \quad n \geq N
$$

with some $c_{1}>0$ independent of $n$ and $h$. Thus, for $0 \leq j \leq N$,

$$
\begin{aligned}
\sum_{k_{1}+\ldots k_{j-1}=n-k} \prod_{i=1}^{j-1} c^{k_{i}} \alpha\left(k_{i}\right) & \leq \sum_{k_{1}+\ldots+k_{j-1}=n-k}\left(c c_{1}\right)^{n-k} \\
& \leq(n-k-1)^{N}\left(c c_{1}\right)^{n-k} \\
& \leq c_{2}^{n-k}
\end{aligned}
$$


for some $c_{2}>0$ independent of $n$ and

$$
\begin{aligned}
\gamma(n) & \leq c_{3} \sum_{j=2}^{N} \sum_{k=0}^{n+1-j} \gamma(k) \sum_{k_{1}+\ldots+k_{j-1}=n-k} \prod_{i=1}^{j-1} c^{k_{i}} \alpha\left(k_{i}\right) \\
& \leq c_{3} \sum_{j=2}^{N} \sum_{k=0}^{n+1-j} c_{2}^{n-k} \gamma(k) \\
& \leq c_{3}(N-2) \sum_{k=0}^{n-1} c_{2}^{n-k} \gamma(k) \\
& \leq \sum_{k=0}^{n-1} c_{4}^{n-k} \gamma(k)
\end{aligned}
$$

for some $c_{4}>0$ independent of $n$ where

$$
c_{3}=\max \left\{\eta_{1} \eta_{2}, \ldots, \eta_{1} \eta_{N}\right\}
$$

Assume that

$$
\gamma(k) \leq c_{4}^{k} 2^{k-1}, \quad \text { for } 1 \leq k \leq n-1
$$

Then,

$$
\begin{aligned}
\gamma(n) & \leq \sum_{k=0}^{n-1} c_{4}^{n-k} \gamma(k) \\
& \leq c_{4}^{n}+\sum_{k=1}^{n-1} c_{4}^{n-k} c^{k} 2^{k-1} \\
& \leq c_{4}^{n} 2^{n-1}
\end{aligned}
$$

Thus we proved by induction that

$$
\gamma(n) \leq 2^{n-1} c_{4}^{n} \leq c_{*}^{n}
$$

for some $c_{*}>0$ independent of $n$ and $h$. The proof is completed.

Corollary 5.1 Assume that $a(u)$ in (3.1) is a polynomial. Then for any $T>0$ and $s \geq 1$, the map $K$ defined by the IVP (3.1) is analytic from $H^{s}(R)$ to $X_{l, r}^{T, s}$ with $(l, r) \in\left[0, s-\frac{3}{4}\right] \times\left[0, s-\frac{3}{4}\right)$. 


\section{References}

[1] J. L. Bona \& R. Scott, Solutions of the Korteweg-de Vries equation in fractional order Sobolev spaces, Duke Math. J., 43(1976), 87 - 99

[2] J. L. Bona \& R. Smith, The initial value problem for the Korteweg-de Vries equation, Roy. Soc. London, Ser. A, 278(1978), 555 - 601

[3] L. Carleson, Some analytical problems related to statistical mechanics, Euclidean Harmonic Analysis, Lecture Notes in Math., SpringerVerlag, 779(1979), $9-45$

[4] A. Cohen, Solutions of the Korteweg-de Vries equation from irregular data, Duke Math. J., 45(1978), $149-181$

[5] A. Cohen \& T. Kappeler, Solution to the Korteweg-de Vries equation with initial profile in $L_{1}^{1}(R) \cap L_{N}^{1}\left(R^{+}\right), S I A M J$. Math. Anal. , 18(1987), $991-1025$

[6] P. Constantin \& J. C. Saut, Local smoothing properties of dispersive equations, J. Amer. Math. Soc. 1(1988), $413-446$

[7] J. Dieudonné, Foundation of Modern Analysis, Academic Press, 1969

[8] D. Henry, Geormetric Theory of Semilinear Parabolic Equation, Lecture Notes in Math., 840, Springer (1981)

[9] W. Craig, T. Kappeler \& W. A. Strauss, Gain of regularity for equations of KdV type, Analyse Non Linéaire, Ann. Inst. Henri Poincaré, $9(1992), 147-186$

[10] C. S. Gardner, J. M. Green, M. D. Kruskal \& R. M. Miura, A method for solving the Korteweg-de Vries equation, Phys. Rev. Letters, 19(1967), $1095-1097$

[11] - - The Korteweg-de Vries equation and generalizations VI. Method for exact solutions, Comm. Pure and Math., 27(1974), 97 133 
[12] J. Ginibre \& G. Velo, Smoothing properties and retarded estimates for some dispersive evolution equations, preprint

[13] J. Ginibre \& Y. Tsutsumi, Uniqueness for the generalized Korteweg-de Vries equations, SIAM J. Math. Anal., 20(1989), 1388 - 1425

[14] J. Ginibre, Y Tsutsumi and G. Velo, Existence and uniqueness of solutions for the generalized Korteweg-de Vries equation, Math Z., 203(1990), $9-36$

[15] T. Kato, Quasilinear equations of evolutions, with applications to partial differential equation, Lecture Notes in Math. 448, Springer (1975), $27-50$

[16] - On the Korteweg-de Vries equation, Manuscripta Math., 29(1979), $89-99$

[17] - On the Cauchy problem for the (generalized) Korteweg-de Vries equations, Advances in Mathematics Supplementary Studies, Studies in Applied Math., 8(1983), $93-128$

[18] T. Kato \& G. Ponce, Commutator estimates and the Euler and NavierStokes equations, Pure Appl. Math., 41(1988), $897-907$

[19] T. Kappeler, Solutions to the Korteweg-de Vries equation with irregular initial data, Comm. P. D. E., 11(1986), $927-945$

[20] C. E. Kenig, G. Ponce \& L. Vega, On the (generalized) Korteweg-de Vries equation, Duke Math. J., 59(1989), 585-610

[21] - Oscillatory integral and regularity of dispersive equations, Indiana University Math. J., 40(1991), $37-69$

[22] - Well-posedness of the initial value problem for the Kortewegde Vries, J. Amer. Math. Soc., 4(1991), $323-347$

[23] - Well-posedness and scattering results for the generalized Korteweg-de Vries equation via the contraction principle, preprint 
[24] - On the generalized Benjamin-Ono equation, preprint

[25] — - Small solutions to nonlinear Schödinger equations, preprint

[26] C. E. Kenig \& A. Ruiz, A strong type $(2,2)$ estimates for the maximal functions associated to the Schrödinger equation, Trans. Amer. Math. Soc., 230(1983), $239-246$

[27] D. J. Korteweg \& G. de Vries, On the change of form of long waves advancing in a rectangular canal, and on a new type of long stationary waves, Philos, Mag., 5, 39(1895), $422-423$

[28] S. N. Kruzhkov \& A. V. Faminskii, Generilized solutions of the Cauchy problem for the Korteweg-de Vries equation, Math. USSR, Sbornik, 48(1984), $93-138$

[29] P. D. Lax, Integrals of nonlinear equations of evolution and solitary waves, Comm. Pure and Appl. Math., 21(1968), $467-490$

[30] R. M. Miura, The Korteweg-de Vries equation: A survey of results, SIAM Review, 18(1976), 412 - 459

[31] R. M. Miura, S. C. Gardner \& M. D. Kruskal, Korteweg-de Vries equation and generalizations, II. Existence of conservation laws and constant of motion, J. Math. Phys., 9(1968), $1204-1209$

[32] G. Ponce \& L. Vega, Nonlinear small data scattering for the generalized Korteweg-de Vries equation, J. Funct. Anal., (1990), $445-457$

[33] R. L. Sachs, Classical solutions of the Korteweg-de Vries equation for non-smooth initial data via inverse scattering, Comm. PDE, 10(1985), $27-89$

[34] J. C. Saut, Sur quelques généralizations de l'équations de Korteweg-de Vries, J. Math. Pures Appl., 58(1979), 21 - 61

[35] J. C. Saut \& R. Temam, Remarks on the Korteweg-de Vries equation, Israel J. Math., 24(1976), $78-87$ 
[36] A. C. Scott, F. Y. Chu \& D. W. McLaughin, The soliton: A new concept in applied sciences, Proc. IEEE, 61(1973), $1443-1483$

[37] A. Sjöberg, On the Korteweg-de Vries equations: existence and uniqueness, J. Math. Anal. Appl., 29(1970), $569-579$

[38] P. Sjölin, Regularity of solutions to the Schrödinger equations, Duke Math. J. , 55(1987), $699-715$

[39] W. A. Strauss, Nonlinear scattering at lower energy, J. Funct. Anal., 41(1981), $110-133$

[40] R. S. Strichartz, Multipliers in fractional Sobolev spaces, J. Math. Mech., 16(1967), $1031-1060$

[41] _- Restriction of Fourier transform to quadratic surface and decay of solutions of wave equations, Duke Math. J., 44(1977), $705-$ 714

[42] S. Tanaka, Korteweg-de Vries equation: construction of solution in terms of scattering data, Osaka J. Math., 11(1974), 49 - 59

[43] R. Temam, Sur un problème non linéaire, J. Math. Pures Appl., 48(1969), $159-172$

[44] Y. Tsutsumi, The Cauchy problem for the Korteweg-de Vries equation with measure as initial data, SIAM J. Math. Anal., 20(1989), 582 588

[45] L. Vega, The Schrödinger equation: pointwise convergence to the initial data, Proc. Amer. Math. Soc., 102(1988), $874-878$

[46] L. Vega, Doctoral Thesis, University Autonoma de Madrid, Spain (1987)

[47] B.-Y. Zhang, Taylor series expansion for solutions of the KdV equation with respect to their initial values, the IMA preprint, Series \# 1015, Aug. 1992 